\title{
MUSCLE CHARACTERISTICS AND BODY COMPOSITION OF NCAA DIVISION I FOOTBALL PLAYERS
}

\author{
Malia Nikole Melvin
}

A thesis submitted to the faculty of the University of North Carolina at Chapel Hill in partial fulfillment of the requirements for the degree of Masters of Arts in the Department of Exercise and Sports Science (Exercise Physiology).

Chapel Hill

2014

Approved by:

Abbie Smith-Ryan

Eric Ryan

Eric Sobolewski 
(C) 2014

Malia Nikole Melvin

ALL RIGHTS RESERVED 


\begin{abstract}
Malia Nikole Melvin: Muscle Characteristics and body composition of NCAA Division I football players.

(Under the direction of Abbie E. Smith-Ryan)

The purpose of this study was to investigate muscle cross sectional area (mCSA) and echo intensity (EI) of the vastus lateralis (VL) and body composition of Division I football players. Sixty-nine players were stratified by position, race, year, and starter status. Muscle CSA and EI were determined from a B-mode ultrasound panoramic scan of the VL using Image-J software. Fat mass (FM), lean mass (LM), and body fat (\%fat) were determined using dual-energy x-ray absorptiometry. Measures of mCSA $(38.7 \pm 6.6$ $\mathrm{cm}^{2}, \%$ fat $(17.9 \pm 4.6 \%), \mathrm{LM}(81.3 \pm 11.8 \mathrm{~kg})$, and FM $(19.5 \pm 8.7 \mathrm{~kg})$ were found to be significantly different across position $(\mathrm{p}<0.05)$, likely due to the differences in positionspecific tasks. Offensive and defensive linemen were not significantly different $(p>0.05)$, and had the greatest mCSA $\left(44.4 \mathrm{~cm}^{2}\right), \%$ fat $(23.4 \%), \mathrm{LM}(96.4 \mathrm{~kg})$ and FM $(30.9 \mathrm{~kg})$ values in comparison to all other positions. No differences $(p=0.161)$ were observed between positions for EI.
\end{abstract}




\begin{abstract}
AKNOWLEDGEMENTS
I would genuinely like to thank everyone who has helped to make this thesis project a success. I would like to thank my advisor, Dr. Abbie Smith-Ryan, for your continued guidance, patience and support throughout my thesis process. I would also like to thank my committee members, Dr. Eric Ryan and Mr. Eric Sobolewski for taking the time to review my document and offering advice, which has improved the quality of my thesis.
\end{abstract}




\section{TABLE OF CONTENTS}

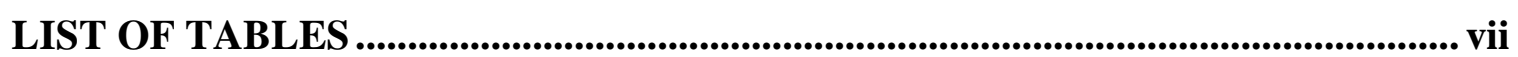

LIST OF FIGURES ................................................................................................. viii

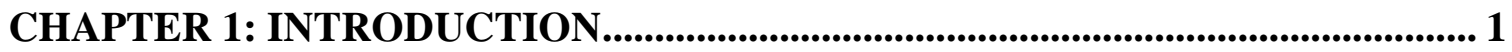

Purpose …............................................................................................................................................. 5

Research Questions................................................................................................................... 5

Research Hypotheses ..................................................................................................... 6

Assumptions ........................................................................................................................................ 6

Theoretical ....................................................................................................................................... 6

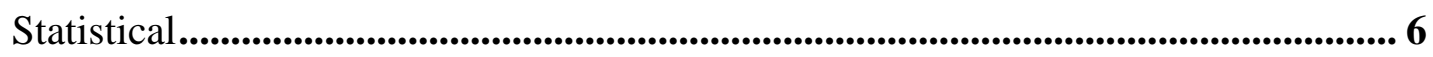

Delimitations ............................................................................................................................. 6

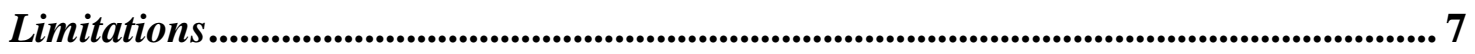

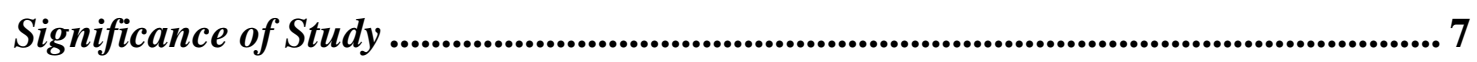

Definitions ........................................................................................................................................ 7

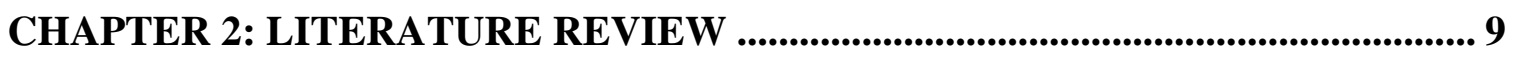

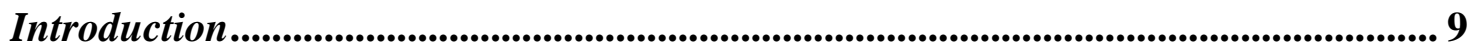

Muscle Quality ..................................................................................................................................... 11

Dual-Energy X-Ray Absorptiometry .................................................................................. 14

Body Composition of American Football Players .............................................................. 17

Significance of Study ....................................................................................................................... 19 
CHAPTER 3: METHODS .................................................................................... 21

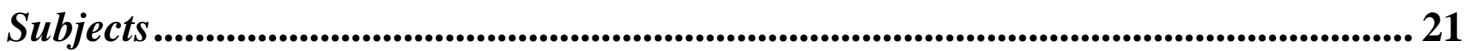

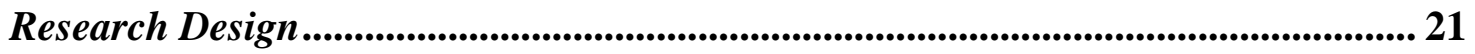

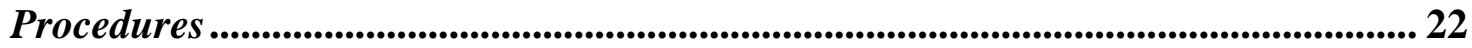

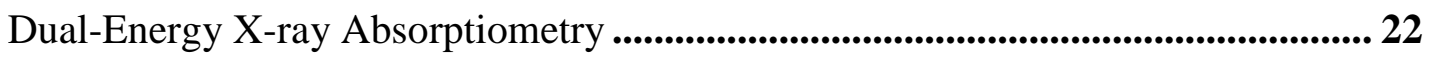

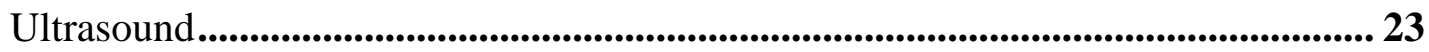

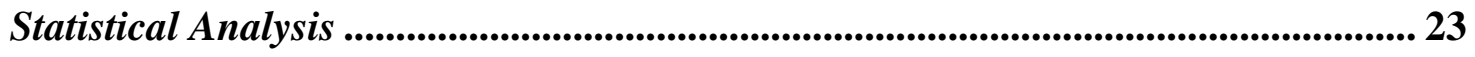

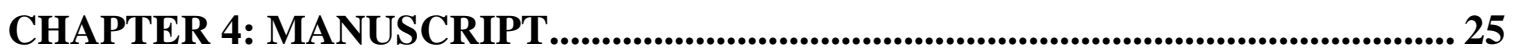

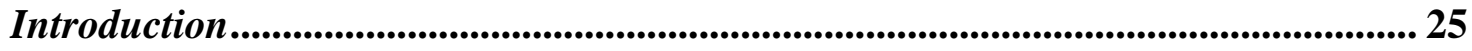

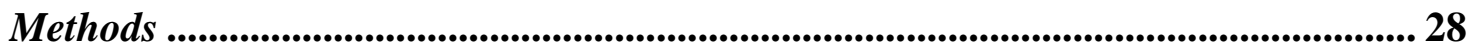

Statistical Analysis .......................................................................................... 31

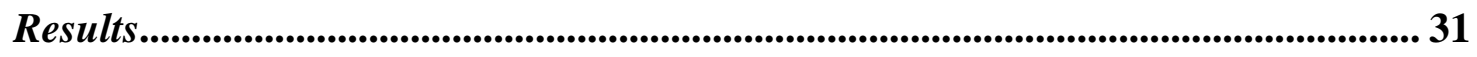

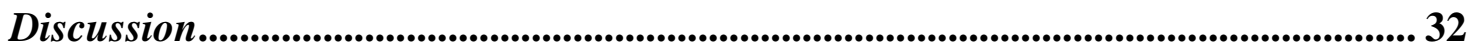

CHAPTER 5: CONCLUSION................................................................................................ 39

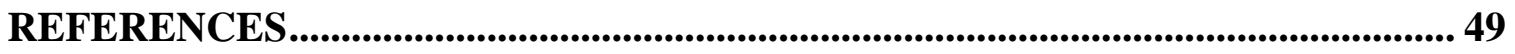




\section{LIST OF TABLES}

Table 1. Mean \pm SD of sample characteristics for position, race, year classification, and starter status subgroups..........................................................41

Table 2. Mean \pm SD of ultrasound characteristics for position, race, year classification, and starter status subgroups...........................................................................................................42

Table 3. Mean \pm SD of DXA body composition characteristics for position, race, year classification, and starter status subgroups................................................43

Table 4. Correlations between US and DXA measures percent body fat (\%fat), lean mass (LM), fat mass (FM), muscle cross sectional area (mCSA),

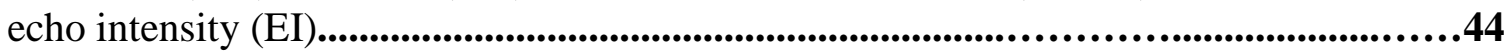




\section{LIST OF FIGURES}

Figure 1. Individual comparisons of height $(\mathrm{cm})$ and weight $(\mathrm{kg})$ stratified

by player position...................................................................................45

Figure 2. Individual echo intensity (a.u.) values stratified by race.............................46

Figure 3. Individual comparisons of lean mass $(\mathrm{kg})$ and fat mass $(\mathrm{kg})$ stratified

by player position.............................................................................47

Figure 4. Individual percent fat values stratified by race...................................................48 


\section{CHAPTER 1}

\section{INTRODUCTION}

The progression of American football, since the 1970s, has been accompanied by a significant increase in player physical size (Kraemer et al., 2005; Noel et al., 2003;

Secora et al., 2004; Snow, Miller-Stafford, \& Rosskopf, 1998). Recent data suggests that players are increasing in weight and speed with a subsequent increase in strength (Robbins et al., 2012). In a study of 37 universities with Division I football teams, Secora et al. (2004) determined that five of the eight player positions increased body weight from 1987 to 2000 (Secora et al., 2004). The study also found that the offensive linemen increased percent body fat (\%fat) significantly, which concurs with most previous studies (Kraemer et al., 2005; Noel et al., 2003; Secora et al., 2004; Snow et al., 1998). Each player position is unique in physical expectations and performance duties; therefore, the body composition of each player on a team may be drastically different depending on position (Kraemer et al., 2005; Mathews \& Wagner, 2008).

An increase in size and body fat of college football players raises concern for future health or performance complications (Albuquerque et al., 2010; Miller et al., 2008; Miller et al., 2002). The World Health Organization reports that overweight and obese individuals are at greater risk of cardiovascular disease, diabetes, and musculoskeletal disorders (World Health Organization, 2013). A study of 261 National Collegiate Athletic Association (NCAA) football athletes reported an inverse relationship between 
\%fat, maximal power clean weight, and vertical jump. Specifically in the offensive and defensive linemen, the 40-yard and 20-yard sprint times were significantly decreased, as \%fat increased (Miller et al., 2002). Therefore, assessing a football athletes' body composition may be beneficial for understanding future health or performance implications.

In addition to the traditional body composition measurements, evaluating \%fat, fat mass (FM) and lean mass (LM), muscle quality measurements may also be advantageous. Muscle quality measurements allow for an enhanced analysis of the muscle tissue by assessing the amount of contractile versus non-contractile tissue within the muscle (Fukumoto et al., 2011). An increase in adipose and connective tissue within the muscle will decrease muscle quality (Cadore et al., 2012; Fukumoto et al., 2011). Goodpaster et al. (2000) determined that intramuscular fat increases with corresponding increases in body fat. Increases in intramuscular adipose and connective tissue may also be related to the development of insulin insensitivity (Goodpaster, 2001). Therefore, as the trend of increasing physical size continues in American football players, it may be important to have a more comprehensive understanding of body composition and muscle quality.

Previous studies have measured football players' \% fat using numerous twocompartment model techniques (Kraemer et al., 2005; Noel et al., 2003; Secora et al., 2004; Snow et al., 1998). A study investigating percent body fat of 36 NFL players using hydrostatic weighing (HW), and 7-site skinfold measures determined that over the course of 20 years, offensive linemen and tight ends had the greatest increase in weight and percent body fat (Snow et al., 1998). Kraemer et al. (2005) investigated body 
composition of 53 NFL players using air displacement plethysmography (ADP) via the BodPod, and found that offensive and defensive linemen have increased body mass most significantly since the 1970s, and that all players were classified as overweight or obese according to body mass index $\mathrm{kg} / \mathrm{m}^{2}$ (BMI) (Kraemer et al., 2005). In 2003, an additional study measured 69 Division I football players using HW and skinfolds, and determined a significant increase in body mass, most notably in offensive and defensive linemen. The increase in body mass was not accompanied by an increase in FFM, which indicates the increased body mass was due to an increase in \%fat (Noel et al., 2003). Mathews et al. (2008) also determined BMI misclassified 55\% of NCAA Division I football players as overweight or obese, in comparison to \% fat from bioelectrical impedance analysis.

Although the various two-compartment methods have been validated by criterion methods, such as hydrostatic weighing, methods for the accurate determination of body composition in an athletic population can be improved upon (Prior et al., 1997). In a racially heterogeneous sample, like a Division I football team, a two-compartment model may not be appropriate. Studies have shown that ADP underestimates \% fat in White males, and overestimates \%fat in a Black population (Sardinha et al., 1998; Wagner et al. 2000). Two-compartment models account for two components of the body, fat mass and fat free mass; density, composition and hydration of fat free mass are assumed constant. Deviations from the assumed values will result in increased systematic error (Prior et al., 1997). Therefore, due to the various body types in an athletic population, measurements of additional compartments have shown to improve the accuracy of body composition measurements (Prior et al., 1997). Multi-compartment methods reduce the standard error of the estimate by accounting for additional components such as total body water, bone 
mineral content and soft mineral content (Z. M. Wang et al., 1998). Dual-energy x-ray absorptiometry is considered a three-compartment model for assessing body composition by quantifying bone mineral content, fat mass, and lean mass. Dual-energy x-ray absorptiometry (DXA) uses low dose radiation and two energy photon beams to assess bone mineral content and soft tissue composition (Nieman, 2010). This multicompartment model is highly correlated to a six-compartment model criterion (Z. M. Wang et al., 1998), and has been shown to be reliable and valid for assessing lean mass for both men and women (Van Loan \& Mayclin, 1992). Therefore, a multi-compartment model should be used to evaluate NCAA Division I football players in order to obtain a more accurate representation of the athletes' current body composition and health status.

As previously mentioned, measures of muscle characteristics may also provide additional information regarding an athlete's health and performance. Muscle characteristics such as muscle cross sectional area (mCSA) and echo intensity (EI) aid in quantifying muscle quality, which estimates the amount of contractile versus noncontractile tissue within the muscle. Echo intensity represents the amount of adipose and connective tissue infiltration within the muscle as measured by a non-invasive grayscale analysis of an ultrasound image. Echo intensity has previously been shown to be related to strength, power, and cardiovascular function (Cadore et al., 2012; Fukumoto et al., 2011). High ultrasound EI measures have been suggested to be related to sarcopenia and sarcopenic obesity (Fukumoto et al., 2011), while increases in intramuscular adipose and connective tissue have also been shown to be influenced by race (Miljkovic et al., 2009). The study of muscle quality in a racially heterogeneous Division I football team may 
provide insight into whether player position or racial group with higher percent body fat are more at risk for health or performance consequences.

\section{Purpose}

1. The purpose of this study was to examine the relationship between muscle characteristics, as measured by muscle cross sectional area and echo intensity of the vastus lateralis, and body composition of NCAA Division I football players.

2. The secondary purpose of this study was to determine if significant differences exist in muscle characteristics of the vastus lateralis and body composition between the following player positions: quarterbacks, running backs, wide receivers, offensive linemen, tight ends, defensive linemen, linebackers, defensive backs, and kickers/punters.

3. Exploratory analyses were performed to determine if significant differences exist in muscle characteristics of the vastus lateralis and body composition between athletes of different races, year classification, and starter status.

Research Questions

$\mathrm{RQ}_{1}$ : Do muscle cross sectional area and echo intensity of the vastus lateralis relate to body composition measurements: percent body fat, fat mass, and lean mass in NCAA Division I football players?

$\mathrm{RQ}_{2}$ : Will vastus lateralis muscle characteristics as measured by mCSA and EI differ between player positions in a NCAA Division I football team?

$\mathrm{RQ}_{3}$ : Will vastus lateralis muscle characteristics as measured by mCSA and EI differ between races, years, or starter status in a NCAA Division I football team? 


\section{Research Hypotheses}

$\mathrm{H}_{1}$ : Muscle cross sectional area of the vastus lateralis will be negatively correlated to percent body fat and fat mass and positively correlated to lean mass; echo intensity of the vastus lateralis will be positively correlated to percent fat and fat mass and negatively correlated to lean mass.

$\mathrm{H}_{2}$ : Vastus lateralis muscle characteristics as measured by mCSA and EI will significantly differ between player positions in a NCAA Division I football team.

$\mathrm{H}_{3:}$ Vastus lateralis muscle characteristics as measured by mCSA and EI will significantly differ between races, year classification and starter status.

Assumptions

Theoretical

- All subjects will be two-hours fasted.

- Muscle quality was accurately measured using echo intensity determined from an ultrasound scan.

Statistical

- Population from which sample was drawn is normally distributed.

- There was a linear relationship between the independent and dependent variables.

\section{Delimitations}

- This study only included NCAA Division I football players.

- Athletes arrived 2-hours fasted.

- Subjects were excluded if they had suffered an injury, which prevented team training within the 3-12 weeks prior to testing. 


\section{Limitations}

- Results may not be generalizable to all athletes as only NCAA Division I football players were measured.

- This study had a small sample size, specifically within each player position.

Significance of Study

This study aimed to determine a relationship between body composition and muscle quality in NCAA Division I football players. This relationship may allow for the determination of whether muscle quality can be used predict \%fat, FM or LM in a group of athletes. Also, an analysis of each player position may help evaluate if any position has decreased muscle quality and an increased risk of health. Exploratory analyses of the athletes' race may also provide beneficial information to determine whether any particular racial group has a decreased muscle quality, which may pre-dispose those individuals to various health risks including metabolic syndrome and obesity-related diseases. This study may prompt the education of Division I athletes about the potential risk of high percent body fat and decreased muscle quality on future health and performance.

\section{Definitions}

Muscle quality: a measure of the amount of contractile versus non-contractile tissue within the muscle (Fukumoto et al., 2012).

Echo intensity: a non-invasive grayscale analysis of ultrasound measures as expressed in values between 0 and 255 a.u. to estimate the amount of intramuscular adipose and connective tissue (Fukumoto et al., 2012). 
Ultrasound (US): a technique used to measure body composition by using a transducer probe to emit, through the skin, an ultrasonic wave, which part is reflected at the fat muscle interface (Pineau, Guihard-Costa, \& Bocquet, 2007).

Dual-Energy X-Ray Absorptiometry (DEXA): a three-component model for estimating body composition that uses low dose radiation to measure bone mineral content, fat mass and lean mass (Nieman, 2010).

Fat mass: all extractable lipids from adipose and other tissues in the body (Heyward, 2001).

Lean mass: non-bone fat free mass, which includes fat free tissues such as water, muscle, connective tissue, and internal organs (Nieman, 2010).

Percent body fat: fat mass expressed as a percentage of total body weight (Heyward, 2001).

Race: an individual's self-identified racial and national origin or sociocultural groups (“About Race," 2012).

White: a person having origins in any of the original peoples of Europe, the Middle East, or North Africa (“About Race," 2012).

Black or African American: a person having origins in any of the Black racial groups of Africa ("About Race," 2012). 


\section{CHAPTER 2}

\section{LITERATURE REVIEW}

\section{Introduction}

Body composition has been shown to significantly influence both exercise performance and health in an array of athletes (Albuquerque et al., 2010; T. a Miller et al., 2002; World Health Organization, 2013). Measurement of body composition attempts to quantify compartments of body mass. Two-compartment models, dividing the body into fat mass (FM) and fat free mass (FFM), are the most common form of measurement used in athletes (Kraemer et al., 2005; Noel et al., 2003; Secora et al., 2004; Snow et al. 1998). However, as body composition analyses have evolved, multi-compartment models have become increasingly popular to obtain a more accurate estimation of body composition (Prior et al., 1997; Wang et al., 2010; Wang et al., 1998).

Measurement of muscle density, as determined by the comparison of the amount of contractile and non-contractile tissue within the muscle, termed muscle quality, has gained interest as a further analysis of muscle tissue. Muscle quality can be assessed by a variety of methods including muscle biopsies (Goodpaster et al, 2001), magnetic resonance imaging (Tracy et al., 1999), computed tomography scan (CT) (Frontera et al., 2000), and ultrasound (Cadore et al., 2012; Fukumoto et al., 2011). In recent literature, the ultrasound has become a common method for analyzing muscle quality by determining the muscles' echo intensity (EI) and muscle cross sectional area (mCSA) (Arts et al., 2010; Cadore et al., 2012; Fukumoto et al., 2011). Echo intensity is an 
estimation of intramuscular adipose and connective tissue, which is measured using a brightness scale of the ultrasound image. Echo intensity measures indicate overall muscle quality by representing the loss in contractile tissue within the muscle as adipose and connective tissue infiltrate the muscle due to aging (Fukumoto et al., 2011) or obesity (Nijboer-Oosterveld et al., 2011).

Current literature has primarily focused on the relationship of muscle quality and aging, sedentary, and recreationally active adults (Cadore et al., 2012; Fukumoto et al., 2011; Sipila \& Suominen, 1991). Studies have also evaluated differences in muscle quality between young and old (Arts et al., 2010), obese and normal weight adults (Nijboer-Oosterveld et. al, 2011), and between races (Miljkovic et al., 2009). However, research investigating muscle quality measures in highly trained individuals is limited, which promotes future research in this population. In obese individuals, an increase in subcutaneous fat mass has been found to be accompanied by a decrease in muscle quality (Goodpaster et al., 2000; Nijboer-Oosterveld et al., 2011) Therefore, athletes with a high percent body fat may demonstrate decreased performance, as a result of a lower muscle quality, although this has not yet been evaluated.

The relationship between muscle quality and body composition has not been given much attention in current literature, but may warrant further investigation due to the potential impact on health and exercise performance. American football players in particular may benefit from a better understanding of muscle quality. Football athletes have a wide variety of body compositions across skill position (Kraemer et al., 2005; Noel et al., 2003). The skill positions are generally stratified by the following: quarterback/kicker/punter, tight end, offensive linemen, running back, wide receiver, 
defensive back, linebacker, and defensive linemen. Five of the eight of the skill positions have seen an increase in body mass and size as football has evolved from the 1980s to the present (Secora et al., 2004). However, the offensive and defensive linemen in particular are the most at risk for increases in percent body fat (Kraemer et al., 2005, Secora et al., 24). This increase in percent body fat, compared to the other football player positions, have been associated with health complications such as obesity, hypertension, sleep disordered breathing, and metabolic syndrome (Albuquerque et al., 2010). Understanding the relationship between muscle quality and body composition in each football player position may provide a better representation of their health risk level due to excess subcutaneous fat and intramuscular non-contractile tissue. This may help these athletes transition from playing into retirement. This literature review will discuss the current relevant research regarding muscle quality, dual-energy x-ray absorptiometry a threecompartment model of body composition, and body composition of American football athletes.

\section{Muscle Quality}

Muscle quality encompasses physiological factors such as the amount of contractile and non-contractile tissue within the muscle and muscle thickness, which influence muscle strength, power and functional capacity (Cadore et al., 2012). Muscle quality can be determined by ultrasound measures of mCSA and EI. Echo intensity has been reported to be a valid non-invasive measure of muscle quality, and has previously been shown to be related to strength, power, and cardiovascular function (Cadore et al., 2012; Fukumoto et al., 2011). Previous data has demonstrated increased intramuscular connective and adipose tissue may result in a lower muscle quality and a higher EI 
measurement (Arts et al., 2010). Therefore, as skeletal muscle fat infiltration increases in elderly and obese individuals, an increased intramuscular fat and EI is anticipated (Miljkovic \& Zmuda, 2010). Increased fat accumulation in the muscle may represent metabolic changes in lipid metabolism including reduced fat oxidation and low basal ATP concentration (Miljkovic \& Zmuda, 2010). Reduced fat utilization will increase excess availability of fat or increase the uptake of lipid into the muscle (Roden, 2005). The increase in intramuscular fat has been shown to be related to insulin insensitivity and the development of metabolic syndrome (Goodpaster et al., 2001). The increase in intramuscular adipose tissue may also represent a decrease in exercise performance (Achten \& Jeukendrup, 2004). It is commonly reported that regular aerobic exercise in healthy (Friedlander et al., 1998 \& 1999) and obese (Van Aggel-Leijssen et al., 2002) individuals increases fat oxidation and is related to endurance capacity and exercise performance (Hawley, Brouns, \& Jeukendrup, 1998).

Acute and chronic training has been shown to improve muscle quality and function in elderly individuals (Sipila \& Suominen, 1991 \& 1993; Tracy et al., 1999). Elderly individuals who are chronically trained, yield higher muscle quality values as a result of reduced connective tissue and less intramuscular fat tissue (Sipila \& Suominen, 1991). Trained individuals also have been shown to have denser quadriceps muscle tissue, less total area of fat, and a decreased relative proportion of fat in the quadriceps muscle (Sipilä \& Suominen, 1993).

Resistance training is particularly important as studies have shown muscle strength to be negatively correlated to EI and positive correlated to muscle thickness (Cadore et al., 2012; Fukumoto et al., 2011; Ikezoe et al., 2012). Fukumoto et al. (2011) 
determined that there is a larger loss of muscle contractile tissue with age, compared to muscle size in the quadriceps. In addition, the study observed that high percent body fat measured in aging adults resulted in sarcopenic obesity, which may be associated with increase intramuscular adipose tissue (Fukumoto et al., 2011). In addition to muscle strength, Cadore et al. (2012) evaluated the relationship between EI and cardiorespiratory fitness. The study determined that there are negative associations between rectus femoris EI and workloads at ventilatory threshold, which suggests connective and adipose tissue infiltration into the muscle may decrease cardiorespiratory capacity. The study hypothesized that as intramuscular adipose and connective tissue increases, it decreases the number of capillaries, which will disrupt the blood supply of the muscle fibers. Therefore, a lower muscle quality may influence aerobic capacity and could be of importance to an athlete of any age.

Trained, younger populations should have a high muscle quality compared to elderly (Arts et al., 2010). However, obesity may have the potential to alter the relationship between age and EI. Studies have suggested that increased percent body fat may be related to a decline in muscle quality (Goodpaster et. al, 20011; NijboerOosterveld et al., 2011). Nijboer-Oosterveld et al. (2011) observed the relationship of muscle quality and obesity in 54 healthy subjects (Sex: 27 males, 27 female; Age range: 21-86), and found that in the rectus abdominis, rectus femoris, and gastrocnemius, EI increased as subcutaneous fat thickness increased. The subjects of greater weight showed increased intramuscular fat. A study in aging adults observed that high percent body fat can result in sarcopenic obesity, which is may be associated with increase intramuscular adipose tissue (Fukumoto et al., 2011). 
The relationship of muscle quality and body composition may be of importance to overweight and obese populations, who are more at risk for development of metabolic syndrome. A study by Goodpaster et al. (2001) performed muscle biopsies in the vastus lateralis of sedentary obese subjects with Type II diabetes, and determined excess accumulation of intramuscular fat was negatively correlated to insulin sensitivity potentially leading to development of Type II diabetes. Another study evaluated the relationship of muscle quality and Type II diabetes using ultrasonography in older Caucasian $($ mean \%fat $=27.2)$ and Afro-Caribbean $($ mean \%fat = 22.2) men $($ Miljkovic et al., 2009). The study concluded that men of African ancestry had significantly greater intramuscular fat than men of Caucasian ancestry, and were at greater risk for development of Type II diabetes. However, body composition measured by DXA found that men of African ancestry had a significantly lower percent body fat than the Caucasian men. This suggests that racial differences may have a greater influence on muscle quality than body composition. The previous studies indicate that future research should investigate the use of ultrasonography in addition to body composition measures as a quick estimation of intramuscular fat as a pre-diagnosis of insulin resistance and type II diabetes (Goodpaster et al., 2001, Nijboer-Oosterveld et al., 2011).

In the current study, as all individuals will be men and highly trained, it will help determined the influence that body composition by player position and race have on an individual's muscle quality.

\section{Dual-Energy X-Ray Absorptiometry}

Body composition and body mass index (BMI) analyses are commonly used to predict possible health complications related to obesity (Gregg et al., 2004; Harp \& 
Hecht, 2005; Lenz, Richter, \& Mühlhauser, 2009). Body composition has also been shown to be a significant predictor of power athletic performance, including vertical jump, maximal power clean weight, and $20 \mathrm{yd}$. and $40 \mathrm{yd}$. sprints (Miller et al., 2002). Due to the limitations of BMI, which does not account for differences in tissue type within the body, and the increased lean body mass of strength-trained athletes, it is not an effective method to assess risk factors for obesity-related health concerns (Hyman, Dang, \& Liu, 2012; Kraemer et al., 2005). Multi-compartment models are considered the criterion method for analysis of body composition, and are used to assess the validity of two-compartment reference body composition methods (Bosy-Westphal et al., 2008; Pateyjohns et al., 2006; Sardinha et al., 1998; Wagner et al., 2000). Multi-compartment models divide the body into its multiple constituents such as fat mass, fat free mass, total body water, bone mineral content, and soft tissue mineral content. Models such as the Siri three-compartment model (Siri, 1961) or the Selinger four-compartment model (Selinger, 1977) require a combination of reference methods including air displacement plethysmography, bioelectrical impedance, and dual-energy x-ray absorptiometry (DXA). However, the DXA divides the body into three-compartments, fat mass, lean mass, and bone mineral content, while assuming constant hydration in the fat free soft tissue (Wang et al., 1998). Therefore, the DXA requires only one, simple reference method to obtain a more accurate multi-compartment body composition analysis.

Dual-energy x-ray absorptiometry uses a stable x-ray generator and two energy photon beams to assess bone mineral content and soft tissue composition by differences of attenuation of the two energy beams (Neiman, 2011). This model divides the body into three components, fat mass, lean mass and bone mineral content. Wang et al. (1998) 
compared various multi-compartment models to a six-compartment (6-C) criterion in 23 healthy individuals (Sex: 17 males, 6 females; Race: 12 White, 3 African American, 9 Puerto Rican). The study determined that the DXA model was highly correlated $\left(r^{2}=0.972\right)$ to the 6-C model for measuring percent body fat, and is therefore, an acceptable and valid method for percent body fat estimations. Additional body composition studies have determined the DXA provides valid measures of percent body fat, as measurements were highly correlated to three-compartment (Norcross \& Van Loan, 2004), four-compartment (Prior et al., 1997), and five-compartment (Wang et al., 2010) models.

Specifically, Prior et al. (1997) investigated body composition assessed by DXA in 172 healthy men and women. The sample included 111 collegiate athletes (Sex: 67 males, 44 females; Race: 39 African American, 72 White) and 61 non-athletes (Sex: 24 males, 37 females; Race: 23 African American, 83 White). Collegiate sports represented included football, basketball, volleyball, gymnastics, swimming and track and field. The results determined that there was no significant difference between DXA percent body fat and the four-compartment model percent body fat estimation, and standard error of the estimate for DXA was $<3 \%$ indicating it is an accurate and valid method for measuring percent body fat in a heterogeneous sample.

The determined validity of the DXA for assessing body composition has led multiple studies to use DXA as the criterion method for cross validation studies (BosyWestphal et al., 2008; Pateyjohns et al., 2006; Sardinha et al., 1998; Wagner et al., 2000). A study by Sardinha et al. (1998) determined percent fat in 62 white men, was systematically underestimated by air displacement plethysmography (ADP) when 
compared to DXA. However, Wagner et al (2000), found that in a sample of black individuals, percent fat measured by ADP was significantly overestimated in comparison to DXA. Therefore, the lack of ability of ADP to detect racial differences is a limitation in assessing percent body fat measures in a racially heterogeneous sample, such as a college football team.

Further studies investigating the validity of bioelectrical impedance body composition measurements in overweight and obese populations found that BIS has acceptable agreement with a DXA criterion (Bosy-Westphal et al., 2008; Pateyjohns et al., 2006). However, a study determined that in 43 overweight and obese men (BMI range: $28-43 \mathrm{~kg} / \mathrm{m}^{2}$ ), bioelectrical impedance was only moderately correlated to percent body fat measures in comparison to DXA $\left(\mathrm{r}^{2}=0.69\right)$ (Pateyjohns et al., 2006). In order to evaluate football athletes body composition, a more comprehensive approach to body composition is recommended as prediction errors for skinfolds, bioelectrical impedance and near-infrared spectrophotometry are much greater when compared to DXA (Collins et al., 1999).

\section{Body Composition of American Football Players}

Body composition of American football players is diverse, as a primary result of position specific performance demands (Kraemer et al., 2005). The body composition characteristics of football players have gained increasing attention due to growing increase in player size and concomitant increase in associated health risks. A study of 36 NFL players, which compared results to a previous study using similar body composition techniques, demonstrated that there was a significant $9 \%$ increase in percent body fat from 1976-1997 of offensive linemen and tight ends (mean \pm SD: $24.7 \pm 4.7 \%$ ) (Snow et 
al., 1998). A study of 37 universities with Division I football programs found that players have continued to increase size from 1990 into the 2000s (Secora et al., 2004). Noel et al. (2003) performed hydrostatic weighing and 7-site skinfold analyses on 69 Division I football players. Percent body fat ranged from $15.2-27.4 \%$ with defensive backs, wide receivers and running backs reporting the lowest percent body fat and offensive and defensive linemen and tight ends reporting the highest. Kraemer et al. (2005) performed air displacement plethysmography analyses on $53 \mathrm{NFL}$ players and saw a similar trend of percent body fat and player position. The team percent body fat ranged from $6.3-25.1 \%$, and again the defensive backs, wide receivers, and running backs had the lowest percent body fat and the offensive linemen had the highest followed by the defensive linemen. Noel et al. (2003) found that when considering fat mass, fat free mass and total body mass, the offensive and defensive linemen have significantly increased in body mass, but not in fat free mass.

Offensive and defensive lineman commonly have the highest percent body fat of the player positions (Kraemer et al., 2005; Noel et al., 2003), and therefore are at the highest risk for development of obesity-related health complications. Retired NFL players, especially offensive and defensive linemen are at greater risk for becoming obese and developing metabolic syndrome, cardiovascular disease, arthritis, and sleep disordered breathing (Albuquerque et al., 2010; Miller et al., 2008). The previous studies demonstrate there is a reason for concern in the player positions with high percent body fat, which may lead to obesity and health complications. 
Significance of Study

The current study aimed to evaluate the relationship between muscle quality and body composition in Division I football players. The study also investigated whether player position or race influence muscle quality and body composition. Muscle quality measures performed using ultrasonography have primarily focused on elderly populations, and body composition values were not evaluated. Therefore, the study results may establish whether the trend in increasing size of football players, especially the offensive and defensive linemen is accompanied by an increase in decreased muscle quality. The comparisons may help to investigate whether a measure of muscle quality in this population can predict skill position or racial subgroups that are at a heightened risk for developing obesity-related health complications such as metabolic syndrome, hypertension and cardiovascular disease. The knowledge acquired may help individuals that are at risk to have a better understanding of their muscle quality due to their percent body fat levels, which may hinder performance, or lead to a more difficult transition into retirement. The ultrasound and echo intensity measurements could therefore be used as a quick and easy measurement to perform in addition to composition measurements to help predict health outcomes in an athletic population.

The results of this study may be of particular importance to future research of retired athletes. Athletes transitioning into retirement may see greater problems depending on player position. Miller et al. (2008) observed in retired NFL players that offensive and defensive linemen have a much higher prevalence of obesity and metabolic syndrome than the other playing positions. However, all of the football players had an increased prevalence of the two previously mentioned diseases in comparison to the 
general population as determined by the National Health and Nutrition Examination Survey. Increased percent body fat and decreased muscle quality have both been seen to be related to the development of metabolic syndrome, and therefore, it is important to have a clear understanding of body composition and muscle quality in this specific population (Goodpaster et al., 2001; Nijboer-Oosterveld et al., 2011). 


\section{CHAPTER 3}

\section{METHODS}

\section{Subjects}

One Hundred and eleven college aged males who were currently participating in NCAA Division I football at the University of North Carolina at Chapel Hill were asked to participate in this study. Of the 111,73 volunteered to participate in the study, and 69 players were used for analysis; $n=4$ were not included due to injury preventing training. Players were stratified by position, which include: quarterbacks (QB), running backs (RB), wide receivers (WR), offensive linemen (OL), tight ends (TE), defensive linemen (DL), linebackers (LB), defensive backs (DB), and kickers/punters (KP). A subsequent stratification by race: black (B), white (W) and other (O; Biracial, Latino, Asian, American Indian); year classification: freshman (Fr), sophomore (So), junior (Jr), and senior (Sr); and by starting status: starter (S) and nonstarter (NS) was performed. Subjects were recruited by verbal recruitment by the investigators during their routine body composition testing. Upon arrival to the Laboratory, all subjects signed the informed consent form approved by the University's Institutional Review Board.

\section{Research Design}

Subjects were asked to report to the laboratory for a 30-minute body composition testing session. Each subject arrived to the laboratory 2-hours fasted. Height was measured using a portable stadiometer (Perspective Enterprises, Portage, MI, USA) and weight was measured using a mechanical scale (Detecto, Webb City, MO, USA). Body 
composition was assessed using dual-energy $\mathrm{x}$-ray absorptiometry. Image-J software was used to quantify echo intensity (EI) and muscle cross sectional area (mCSA) of the vastus lateralis (VL) which were determined from a panoramic scan of the vastus lateralis (VL) using a GE Logiq-e B-mode ultrasound (US).

\section{Procedures}

\section{Dual-Energy X-ray Absorptiometry}

Whole body composition was assessed using a Hologic Dual-Energy X-ray Absorptiometer (DXA, Hologic Discovery W, Bedford, MA) using the device's default software (Apex Software Version 3.3). The DXA uses two, beam energies which produce varying degrees of attenuation as they pass through different masses and types of tissue, and therefore, can determine fat mass, lean mass, bone mineral content, and percent body fat. The device was calibrated according to manufacturer's instructions before testing to ensure valid results. A trained technician performed all scans. The subject were asked to remove all metal, thick clothing, and heavy plastic, which could interfere with the DXA scan. Each subject's identification number, age, ethnicity, height and weight were entered into the DXA software prior to scanning. Subjects were then asked to lay supine in the middle of the scanning platform with hands pronated and arms and legs to the side not touching any other part of their body. However, when necessary a subject's thumbs were placed under the buttocks in order to stay within the platform's width restriction. Subjects were instructed to remain still and breathe normally for the duration of the scan. The scans were automatically analyzed by the software, but specific regions of interest were confirmed by the technician. 


\section{Ultrasound}

Muscle cross sectional area of the vastus lateralis (VL) was determined using a GE Logiq-e B-mode ultrasound (GE Healthcare, Wisconsin, USA) from a panoramic scan of the thigh. The wide-band linear array ultrasound transducer probe (GE: 12L-RS) was held perpendicular to the tissue and swept across the skin at equal pressure from the lateral VL border to medial fascia separation. The same technician performed each scan while the subjects laid supine with the right leg extended and relaxed on the examination table. Echo intensity was also determined from the panoramic scan of the VL by grayscale analysis using Image-J software (National Institute of Health, USA, Version 1.37). As previously described by Cadore et al. (2012), the same technician traced the outline of the VL for each subjects' scan along the fascia border as close as possible to capture only the muscle.

\section{Statistical Analysis}

All statistical analysis were performed using SPSS Version 20 Statistical Analysis Software (IBM, Somers, NY, USA). Descriptive statistics (mean \pm standard deviation) including, height, weight, percent body fat (\%fat), echo intensity (EI), muscle cross sectional area (mCSA), fat mass (FM), and lean mass (LM) were performed for all subjects. In addition, a one-way analysis of variance was performed to evaluate position and racial comparisons of \%fat, EI, mCSA, FM, LM. For position comparisons the sample included quarterbacks, running backs, wide receivers, offensive linemen, tight ends, defensive linemen, linebackers, defensive backs, and kickers/punters. For racial comparisons the sample included white, black and other; for year classification the sample included freshman, sophomore, junior and senior; for starter status the sample 
included starter and non-starter. If differences were observed a Bonferroni post-hoc was performed. Pearson's correlation coefficients were calculated between EI, mCSA, \%fat, FM, LM and Legs LM. An alpha level of $\mathrm{p} \leq 0.05$ was established a priori. 


\section{CHAPTER 4}

\section{MANUSCRIPT}

\section{Introduction}

An American football team is composed of athletes with various physiological characteristics, largely due to different position specific demands. Recent data evaluating differences in football players across position, level (i.e. collegiate v. professional), and ability (i.e. drafted v. undrafted) has expanded in an effort to better understand the characteristics of a successful elite athlete, as well as identify potential risk for disease. Body size and composition have been shown to be primary contributors to performance (Miller et al., 2002). Additionally, while most football athletes have shown a trend toward increasing body mass, there has been a concomitant increase in fat free mass (Kraemer et al., 2005). Evaluating baseline differences across position may be useful to reference if a player suffers an injury, gains or loses weight, or declines in performance. Measurement of body composition and the quality of the muscle may allow for a better identification of current and future health risks in these athletes. A focus on the collegiate football athlete is important, as many of these players will not go on to play professionally, and the athletes who do, have an average career length of 3.5 years according to the National Football League Communications ("What is average NFL player's career," 2011). 
Previous studies have measured percent body fat (\%fat) of football athletes using numerous two-compartment model techniques including hydrostatic weighing, 7-site skinfolds, bioelectrical impedance and air displacement plethysmography (Kraemer et al., 2005; Mathews \& Wagner, 2008; Noel et al., 2003; Secora et al., 2004; Snow et al. 1998). While two-compartment methods are widely utilized, and validated, due to the various body types in an athletic population, measurements of additional compartments have shown to improve accuracy of body composition (Prior et al., 1997). Dual-energy xray absorptiometry is a three-compartment model that assesses body composition by quantifying bone mineral content, fat mass, and lean mass, and is highly correlated to a six-compartment model criterion (Wang et al., 1998). In previous literature, offensive and defensive linemen have commonly reported the highest \%fat (Kraemer et al., 2005; Noel et al., 2003; Snow et al., 1998), and therefore may be at a greater risk for development of obesity-related health complications (Kopelman, 2007). Retired NFL players, especially offensive and defensive linemen, have an increased risk for becoming obese and developing metabolic syndrome, cardiovascular disease, arthritis, and sleep disordered breathing (Albuquerque et al., 2010; Miller et al., 2008). A recent study of 68 NFL retirees found metabolic syndrome was present in $50 \%$ of the individuals, highlighting the increased risk for health complications in this population (Kelly et al., 2014). In addition, a study of 261 NCAA football athletes reported an inverse relationship between \% fat, maximal power clean weight, and vertical jump, emphasizing the negative impact higher body fat may have on reduced performance (Miller et al., 2002). Therefore, an accurate measure of body composition may be a valuable tool for players, strength 
coaches, and sports nutritionists in order to prescribe successful training and nutrition interventions to allow individuals to reach their maximal athletic potential.

In addition to the traditional body composition measurements, determining muscle characteristics may also be advantageous. In recent literature, ultrasonography has become a common method for analyzing muscle quality by determining muscle echo intensity (EI) and muscle cross sectional area (mCSA) (Arts et al., 2010; Cadore et al., 2012, Fukumoto et al., 2011). Echo intensity, which is measured using a brightness scale of an ultrasound image, may indicate muscle quality by estimating greater intramuscular fat and/or connective tissue due to factors such as aging (Fukumoto et al., 2011) and obesity (Nijboer-Oosterveld et. al, 2011). Muscle quality and size have been shown to be related to muscle strength, power, and functional capacity (Cadore et al., 2012; Fukumoto et al., 2011).

As the trend of increasing physical size continues in American football players, it may be important to have a more comprehensive understanding of body composition and muscle quality in these athletes. Previous research has determined differences in body composition and muscle characteristics across football position (Kraemer et al., 2005), year (Jacobson et al., 2013), athletic success (Miller et al., 2002), and race (Goodpaster et al., 2001). Further investigations of an American football team will help evaluate if any subgroup has an increased risk of performance or health complications. This may help to develop various training and nutrition strategies during competition, as well during the transition to retirement. Evaluating the relationship between muscle characteristics and body composition in highly trained individuals is limited in existing current literature. This relationship may warrant further investigation due to the potential impact on health 
and exercise performance. Therefore, the primary purpose of the current study was to examine the relationship between muscle characteristics of the vastus lateralis and body composition of NCAA Division I football players. Differences in muscle characteristics and body composition between player position, race, year, and starter status were also evaluated.

\section{Methods}

Subjects

Seventy-three NCAA Division I football players volunteered to participate in this study; of those, 4 were removed due to prior injury, which may have influenced muscle quality and body composition measurements. Therefore, 69 NCAA Division I football players (Mean \pm SD; Age: $20.0 \pm 1.1$ yrs; Height: $186.2 \pm 7.0 \mathrm{~cm}$; Body mass: $106.3 \pm$ $21.1 \mathrm{~kg}$; \%fat: $17.9 \pm 4.6)$ were evaluated in this study. Prior to testing all subjects signed an informed consent approved by the University's Institutional Review Board for the protection of human subjects. Subjects were stratified by player position: quarterbacks (QB), running backs (RB), wide receivers (WR), offensive linemen (OL), tight ends (TE), defensive linemen (DL), linebackers (LB), defensive backs (DB), and kickers/punters (KP). Subjects were also stratified by race: black (B), white (W) and other (O; Biracial, Latino, Asian, American Indian); classification: freshman (Fr), sophomore (So), junior (Jr), and senior (Sr); and by starter status: starter (S) and nonstarter (NS). Group descriptive characteristics are presented in Table 1 and Figure 1. Experimental Design

The present study was completed in one 30-minute visit. Subjects arrived to the laboratory 2 hours fasted and after abstaining from exercise for a minimum 2 hours. 
Upon arrival, height (Perspective Enterprises, Portage, MI) and weight (Detecto, Webb City, MO, USA) were measured, and an exercise and diet status questionnaire was given to ensure pre-assessment guidelines were met, as well as to account for any existing injury that might influence measurement outcomes. Muscle cross sectional area (mCSA) of the vastus lateralis (VL) was determined from a panoramic scan of the VL using a Bmode ultrasound. Echo intensity was determined offline using Image-J software. Dualenergy x-ray absorptiometry (DXA) was used to determine percent body fat (\%fat), fat mass (FM), and lean mass (LM).

Ultrasound Measurements

Muscle cross sectional area and EI of the VL was determined from a panoramic scan of the thigh using a GE Logiq-e B-mode ultrasound device (GE Healthcare, Wisconsin, USA). The ultrasound settings (Frequency: $26 \mathrm{~Hz}$, Gain: 68, Depth: $4.5 \mathrm{~cm}$ ) were kept constant to standardize mCSA and EI measures. In the instance that the depth was not sufficient to show the entire fascia border, the amount of visible muscle was analyzed to determine EI, and an additional scan was performed at a greater depth to determine mCSA. Prior to the ultrasound measures, subjects were asked to lay supine for 3-5 minutes. During the measure, the subjects laid supine with the right leg extended and relaxed on the examination table with a high-density foam pad strapped to the midpoint of the thigh. To obtain the panoramic scan, a wide-band linear array ultrasound transducer probe (GE: 12L-RS) was held perpendicular to the tissue and swept across the skin at equal pressure from the lateral VL border to medial fascia separation. The same technician (MNM) performed each scan. Echo intensity and mCSA were determined from the same panoramic scan of the VL using Image-J software (National Institute of 
Health, USA, Version 1.37). EI was determined in the standard histogram function, which uses grayscale analysis of pixels ranging from 0 to 255 . Prior to measuring mCSA and EI, each image was calibrated by measuring the number pixels within a known distance of $1 \mathrm{~cm}$. To measure mCSA and EI, as previously described by Cadore et al. (2012), the same technician (MNM) traced the outline of the VL for each subjects' scan along the fascia border as close as possible to capture only the muscle. Test-retest reliability for EI and mCSA measurements taken from a previous study in this lab for individuals of similar stature demonstrated an ICC of 0.74 and 0.87 and SEM of 4.58 a.u and $2.12 \mathrm{~cm}^{2}$, respectively. Dual-energy X-ray Absorptiometry (DXA)

Each subject completed a full body scan using the DXA (Hologic Inc., Bedford, MA, USA; Apex Software Version 3.3) to determine lean mass (LM), fat mass (FM), and percent body fat (\%fat). Each scan was performed by a trained DXA technician (ASR). Prior to testing, subjects were asked to remove all metal, thick clothing, and heavy plastic to reduce interference from the scan. Birthdate, height, weight and ethnicity were entered into the computer. Subjects were placed supine in the center of the scanning table; if the participants' shoulders were too wide to fit in the area of the scan, thumbs were tucked under their buttocks to capture the full scan. Additionally, if participants' height did not allow their entire body to be scanned $(n=8)$, the tips of toes were not included in the scan. Test-retest reliability from a previous study in individuals of similar stature for \%fat, has been reported as $\mathrm{ICC}=0.964$ and $\mathrm{SEM}=1.279 \%$. 


\section{Statistical Analysis}

Separate one-way analyses of variance (ANOVAs) were used to evaluate differences between position, race, year classification, and starter status comparisons for muscle characteristics (EI, mCSA) and body composition (\%fat, FM, and LM). Pearson's correlation coefficients were calculated between EI, mCSA, \%fat, FM, LM and Legs LM. All analyses were run using SPSS (Version 20, IBM, Somers, NY, USA).

\section{Results}

Ultrasound measures of mCSA and EI for each subgroup of position, race, year classification, and starter status are presented in Table. 2. For mCSA, DL ( $46.7 \pm 4.2$ $\left.\mathrm{cm}^{2}\right)$ had significantly greater $(\mathrm{p}=0.000-0.037)$ area than $\mathrm{WR}, \mathrm{LB}, \mathrm{DB}, \mathrm{PK}$, and $\mathrm{RB}(32.3$ $\left.\pm 5.7-41.3 \pm 1.7 \mathrm{~cm}^{2}\right)$ (Table 2). Muscle CSA for OL $\left(42.0 \pm 5.5 \mathrm{~cm}^{2}\right)$ was significantly greater $(\mathrm{p}=0.003)$ than WR $\left(32.3 \pm 5.7 \mathrm{~cm}^{2}\right)$. Echo intensity values revealed no significant differences $(\mathrm{p}=0.161)$ between positions (Table 2$)$. Furthermore, there were no significant differences $(\mathrm{p}=0.063-0.864)$ between race, year classification, or starter status for CSA, and EI measures (Table 2). However, EI between black (57.5 \pm 6.4 a. u.) and white (61.0 \pm 6.7 a. u.) approached significance $(\mathrm{p}=0.063)$ (Figure 2).

DXA Body Composition Characteristics

Body composition variables including \%fat, LM, and FM for each subgroup are included in Table 3. For \%fat, there was no significant difference ( $p>0.05)$ between OL $(22.3 \pm 2.3 \%)$ and DL $(24.4 \pm 2.2 \%)$, but both positions were significantly greater $(\mathrm{p}<0.001-0.021)$ than WR, LB, DB, PK, and RB (13.9 - 17.0\%). Quarterbacks $(18.1 \pm$ $2.1 \%)$ were found to have significantly less \%fat $(\mathrm{p}<0.001)$ than OL, but significantly greater $(\mathrm{p}=0.049)$ than DB $(\Delta 4.1 \%)$. For LM, there was no significant difference 
(p>0.05) between OL $(96.6 \pm 6.8 \mathrm{~kg})$ and DL $(96.2 \pm 4.8 \mathrm{~kg})$, but both positions were significantly greater $(\mathrm{p}<0.008)$ than all other player positions. In addition, LB and TE were not significantly different $(\mathrm{p}>0.05)$ from each other, but were significantly greater $(\mathrm{p}<0.020)$ than WR, DB, and PK. For FM, similarly to \% fat and LM, there was no significant difference ( $p>0.05$ ) between OL and DL, but both positions were significantly greater $(\mathrm{p}<0.003)$ than $\mathrm{QB}, \mathrm{WR}, \mathrm{LB}, \mathrm{DB}, \mathrm{PK}$, and $\mathrm{RB}$. However, only OL were found to be significantly greater $(29.9 \pm 9.4 \mathrm{~kg} ; \mathrm{p}=0.018)$ than TE $(19.4 \pm 4.0 \mathrm{~kg})$. Individual comparisons between LM and FM within each position are presented in Figure 3. It was also determined that for combined leg lean mass (LLM), and trunk fat (TF), there were no significant differences $(\mathrm{p}<0.05)$ between OL and DL, however, for LLM both positions were significantly greater $(\mathrm{p}<0.046)$ than $\mathrm{WR}, \mathrm{LB}, \mathrm{DB}$, and PK; additionally $\mathrm{TF}, \mathrm{OL}$ and DL had significantly greater LLM $(\mathrm{p}<0.003)$ than all other positions. Similarly, to ultrasound measures, there were no significant differences $(\mathrm{p}=0.074-0.983)$ between race, year classification, or starter status for DXA body composition variables. However, \%fat between black and white individuals approached significance $(\mathrm{p}=0.074)$ (Figure 4).

Correlations Analysis

Significant positive correlations were observed between mCSA and \%fat, LM, FM, and LLM ( $r=0.388-0.632, \mathrm{p}<0.01)($ Table 4$)$. Muscle CSA was significantly negatively correlated to $\mathrm{EI}(\mathrm{r}=-0.455, \mathrm{p}<0.01)$. However, there were no significant correlations between EI and DXA values.

\section{Discussion}

Several studies have established that the physical and health risks for collegiate and professional football players are high (Albuquerque et al., 2010; Miller et al., 2008; 
Olson et al., 2011). Health concerns have been reported to be acute and chronic depending on the nature of the position or physical preparedness of the individual player. A chronic health concern, particularly with lineman, is related to metabolic syndrome (Miller et al., 2008); however there is not sufficient research to support a direct link for all football players. Body composition analyses have been determined to be an appropriate predictor of future metabolic concerns including insulin insensitivity, Type II diabetes, and sleep disordered breathing (Albuquerque et al., 2010). In addition to body composition, muscle quality measures may also be possible indicators of metabolic function and performance (Achten \& Jeukendrup, 2004, Goodpaster et al., 2001, NijboerOosterveld et al., 2011). There is limited research on muscle quality norms within this population, and therefore, it is not known how mCSA and EI may influence a collegiate athlete's performance or future health. Similarly, there is little data known about the body composition of Division I football players due to the inconsistency of devices used within each study. Previous research analyzing body composition of football players has theorized that OL and DL have high \% fat due to large structure and the demands of the position. However, the device used for analysis may significantly influence the "at-risk" categorization of lineman. The results of the current study demonstrate that OL and DL were similar in body composition, and both were significantly greater than almost all other positions in \%fat (Mean Difference: $8.7 \pm 0.25 \%$ ), FM (MD: 17.5 $\pm 1.6 \mathrm{~kg}$ ), and LM (MD: $21.8 \pm 0.48 \mathrm{~kg}$ ). According to the data in this study, which determined \%fat of OL and DL to be $24.4 \pm 2.2 \%$ and $22.3 \pm 2.3 \%$, respectively, placing them between the $20^{\text {th }}-25^{\text {th }}$ percentile for other men their age (Body Composition Data, 2010). In comparison, a recent study by Dengel et al. (2014) reported \%fat of OL and DL in professional NFL 
players to be $28.8 \pm 3.7 \%$ and $25.5 \pm 7.6 \%$, respectively placing them between the $50^{\text {th }}-75^{\text {th }}$ percentile. This may suggest that players in this position are susceptible to increased health risks. However, the use of DXA as a method of analysis should be taken into consideration, as DXA may over predict \%fat measures in individuals with a large stature, consisting primarily of LM. Furthermore, the players in the present study demonstrated lower \%BF in comparison to Dengel et al. (2013), likely as a result of varied DXA models (Hologic vs. Lunar, respectively).

To the best of our knowledge, there has been no previous research investigating muscle quality and mCSA of competitive football athletes. Previous studies in young and old populations have demonstrated a wide range of mCSA and EI values. However, direct comparison of values between studies may not be beneficial due to the variation in US model, technique, settings, and muscles used for analyses. However, values for EI from the current study were much lower (59.3 \pm 6.6 a.u.) than previous studies (71.6-129.9 a.u.). Due to the training demands of DI football, a lower EI value is not surprising, thereby representing a better muscle quality. Previous studies have demonstrated an increase in muscle size (Tracy et al., 1999), and improvement in muscle quality, as a result of strength training (Sipila and Suominen, 1991). Sipila and Suominen (1993) determined that 18 weeks of strength training significantly decreased EI in older women, indicating an improvement in muscle quality. Additional studies by Fukumoto et al. (2011) and Cadore et al. (2012) reported EI ranges of 75.3-129.9 a.u. from the quadriceps muscle in elderly men and women using B-mode GE ultrasound devices, without consistent depth and gain settings. A recent study by Rosenberg et al. (2013) reported EI values of $71.55 \pm 4.97$ a.u from 16 male recreationally active participants, using similar 
gain and depth settings to the current study. Measures of muscle quality may be of importance when evaluating overweight football players, especially OL and DL. Although these individuals had significantly greater \%fat in the current study, their muscle quality was not significantly different in comparison to other positions. This may suggest that athletes with higher \%fat may have a better muscle integrity and less intramuscular fat and connective tissue in comparison to untrained overweight individuals (Nijboer-Oosterveld et al., 2011).

A study investigating mCSA of the VL in untrained men reported mean values of $26.7 \pm 4.5 \mathrm{~cm}^{2}$ (Ahtiainen et al., 2010). After 21 weeks of resistance training, mCSA significantly increased to $30.5 \pm 5.7 \mathrm{~cm}^{2}$. Sipila and Suominen (1993) also determined there was a trend of increased mCSA of the quadriceps in older female athletes in comparison to untrained older women $\left(\Delta 4.8 \mathrm{~cm}^{2}\right)$. Previous mCSA values reported in healthy untrained adults (mCSA: $19.8 \pm 1.9 \mathrm{~cm}^{2}$ ) (Scott et al., 2012) were considerably lower than compared to older female resistance trained athletes (mCSA: $31.5 \pm 6.2 \mathrm{~cm}^{2}$ ), (Sipila and Suominen, 1996) and the competitive football players in the current study $\left(38.7 \pm 6.6 \mathrm{~cm}^{2}\right)$. Resistance and anaerobic training promotes the synthesis and growth of contractile proteins which results in whole muscle hypertrophy, as well as hypertrophy within the myofibers (Deschenes \& Kraemer, 2002). Chronic resistance training increases the percentage of type IIA fibers, which have been shown to experience the greatest amount of hypertrophy in comparison to type IIB and type I fibers (Deschenes and Kraemer, 2002). Therefore, greater mCSA is anticipated due to the high volume of resistance training DI football players undergo. Players in the current study with significantly more total lean mass (OL and DL) also demonstrated significantly greater 
mCSA values (DL: $46.7 \pm 4.2 \mathrm{~cm}^{2}$; OL: 42.0 $\pm 5.5 \mathrm{~cm}^{2}$ ). Interestingly, QB, RB, and TE had comparable mCSA values $\left(38.7 \pm 5.2 \mathrm{~cm}^{2}\right)$, however, this was likely due to small position specific sample size, which was a limitation of the study. Ultrasound measures of muscle quality (MQ) and mCSA may provide beneficial information for tracking changes that occur during season, as well as during off-season training. US measures may have the ability to depict muscle integrity imbalances, and potentially be a useful tool for injury prevention. The limb lean and fat mass measures provided by the DXA, in addition to mCSA, and EI US measures, may represent a more clear analysis of how season, training, or an injury has impacted the athlete's muscle composition. These noninvasive measures of MQ and size may also provide a better interpretation of health classification for athletes with greater amounts of body fat. For example, in the current study, although the OL and DL had BMI and \%fat values that classified them 'at risk', their MQ values were similar, if not better, than most other positions. Future research expanding normative values to athletes regarding MQ comparisons may provide greater utility for the US as a tool for health classification of athletes.

While it is apparent that size and body composition vary dramatically within and between football players due to position specific demands, limited data exists regarding their stature (Kraemer et al., 2005). Noel et al. (2003) determined collegiate football players' percent body fat values using hydrostatic weighing (HW) and skinfolds ranged from 15.2-27.4\%. Collins et al. (1999) reported percent body fat of collegiate football players from HW to be $10.7-23.5 \%$. Bioelectrical impedance analysis has also been used to determine percent fat values in this population with values ranging from $13.2-27.6 \%$ (Mathews \& Wagner 2008). Consistency among available data demonstrate DB, WR, and 
RB tend to have the lowest percent body fat, regardless of testing method, while OL and DL have the highest (Kraemer et al., 2005; Noel et al., 2003; Snow et al., 1988). The percent fat values found in this study (13.9-24.4\%) were within the expected ranges in comparison to previous research. However, each of the previously mentioned studies used varying techniques for body composition analysis, and therefore makes individual comparisons more difficult. Although DXA, as used in the current study, has been shown to be highly correlated to criterion multi-compartment models (Norcross \& Van Loan, 2004; Prior et al., 1997; Wang et al., 1998, 2010), depending on the manufacturer (Hologic vs. Lunar), the DXA may slightly over predict percent body fat in an athletic population (Moon et al., 2007; Prior et al., 1997; Wang et al., 1998). However, the ability of the DXA to provide regional lean and fat mass may be beneficial for athletes to assess the specific areas of the body that may be of concern. When returning to play from an injury, the DXA and US measures may be beneficial for quantifying muscle imbalances, particularly between each arm and leg. In addition, when prescribing weight loss or gain interventions, quantifying regional distributions of fat, as well determining muscle imbalances, may improve resistance exercise prescriptions. Future research investigating the influence of a football season on regional lean mass may be valuable especially for resistance training and conditioning prescriptions.

In addition to investigating differences between athletes by position, the current study also stratified athletes by year, starter status, and race. Theoretically, upperclassmen who have regularly participated in a collegiate strength training program, and starters, should have greater lean mass than underclassmen and non-starters (Jacobson et al., 2013). A recent study in Division I football players by Jacobson et al. 
(2013) determined that in 4 years, OL, DL, DB, and WR increased body mass and strength, while OL and DL decreased \%fat, and DB and WR maintained \%fat values. However, there were no significant differences found between athletes' classified year, or starter status, in the current study. Therefore, it may be valuable to assess longitudinal changes that occur within each individual as they progress through their collegiate career. Previous studies have also found significant differences in intramuscular fat and muscle composition between black and white individuals (Ama et al., 1986; Miljkovic et al., 2009); in contrast no significant racial differences were observed in the current study. Similar results were reported by Abe et al. (1999) who found no muscle architectural differences in muscle thickness, pennation angle, and fascicle length of the VL between black and white collegiate football players. This may suggest that chronic resistance training and conditioning may overcome racial differences of MQ in athletes, or it could be related to the homogeneity of our population (i.e. all football). Although not statistically significant, there may be a clinical significance of \%fat measures stratified by race (W: $19.3 \pm 4.0 \%$; B: $16.6 \pm 4.8 \%)$.

The current study demonstrated a significant relationship between DXA body composition values and mCSA, suggesting a strong link between muscle size and DXA derived composition values. However, while a significant negative correlation between mCSA and EI was determined, EI was not correlated to any DXA body composition variables. This suggests that mCSA measures may be related to body composition in athletes. 


\section{CHAPTER 5}

\section{CONCLUSION}

In conclusion, ultrasound measures of mCSA and DXA values of \%fat, LM, FM, LLM were significantly different across position, and therefore may provide beneficial reference information when adapting training or nutrition regiments as a result of season, weight goals, or injury. No significant differences across position were found for EI. This may indicate that athletes of higher \%fat, such as the DL and OL are able to maintain muscle integrity, reducing the concern for immediate performance or health complications that is predicted by body composition measures alone. Recognizing muscle imbalances with US or DXA measures may also improve the quality of resistance exercise prescription, and help determine whether an injured athlete is ready to return to play. As US devices are readily available in most athletic facilities as a clinical tool, it may provide other practical measures for athletes, athletic trainers, and strength coaches. The addition of mCSA and EI, combined with body composition values may be important for athletes considered "at risk" due to \% fat values or injury status. In addition, no significant differences were found between races, years or starter status. Future research investigating the relationship of US and DXA measures stratified by race, year and starter status within each position, using a larger sample of collegiate football players, may produce significant data. Quantifying differences across race, year and starter status may provide insight into the effectiveness of strength and conditioning programs, as well as help determine the characteristics of elite, successful athletes within 
each position.

The small sample size, specifically within each player position, is a limitation of the current study. Additional limitations of the current study included the limited fasting time before the DXA scan ( $\geq 2$ hours) and, due to time restraints, subjects only rested 3-5 minutes before the US scan to account for fluid shifts. However this data expands the small existing body of literature that quantifies the physiological profile of NCAA Division I football athletes. Future research, in both collegiate and professional football athletes, is needed in order to better quantify their health, prevent injury, and appropriately adapt their training. 
Table 1. Mean \pm SD of sample characteristics for position, race, year classification, and starter status subgroups.

\begin{tabular}{|c|c|c|c|c|}
\hline Group & N & Age & Height (cm) & Weight (kg) \\
QB & 5 & $19.6 \pm 0.9$ & $186.6 \pm 2.7$ & $96.9 \pm 2.1$ \\
RB & 4 & $20.5 \pm 1.7$ & $178.0 \pm 5.0^{*}$ & $94.4 \pm 10.2$ \\
TE & 4 & $20.0 \pm 0.8$ & $191.7 \pm 5.0$ & $109.7 \pm 6.6$ \\
LB & 7 & $19.9 \pm 0.9$ & $187.5 \pm 3.4$ & $103.5 \pm 3.9$ \\
DB & 15 & $19.9 \pm 1.1$ & $180.7 \pm 2.8^{*}$ & $89.4 \pm 6.3$ \\
PK & 3 & $20.0 \pm 1.0$ & $176.2 \pm 2.5^{*}$ & $87.5 \pm 0.7$ \\
DL & 9 & $20.1 \pm 1.2$ & $190.6 \pm 4.8$ & $132.2 \pm 9.4^{+}$ \\
OL & 12 & $20.2 \pm 1.3$ & $195.0 \pm 4.2$ & $136.5 \pm 11.0^{+}$ \\
WR & 10 & $19.9 \pm 1.1$ & $183.1 \pm 5.2^{*}$ & $88.0 \pm 6.6$ \\
& & & & \\
Black & 36 & $19.9 \pm 1.2$ & $185.3 \pm 6.0$ & $104.1 \pm 20.1$ \\
White & 26 & $20.0 \pm 1.0$ & $186.7 \pm 8.2$ & $107.1 \pm 21.6$ \\
Other & 8 & $20.6 \pm 0.5$ & $188.2 \pm 6.5$ & $113.2 \pm 24.0$ \\
& & & & \\
Freshman & 7 & $18.3 \pm 0.5$ & $181.9 \pm 8.1$ & $105.0 \pm 27.6$ \\
Sophomore & 33 & $19.7 \pm 0.8$ & $186.8 \pm 6.5$ & $105.8 \pm 20.4$ \\
Junior & 20 & $20.6 \pm 0.5$ & $186.0 \pm 6.2$ & $105.9 \pm 21.1$ \\
Senior & 9 & $21.2 \pm 1.0$ & $188.1 \pm 9.1$ & $110.1 \pm 22.1$ \\
& & & & \\
Starter & 23 & $20.3 \pm 1.0$ & $187.2 \pm 7.2$ & $109.4 \pm 21.0$ \\
Non-Starter & 46 & $19.9 \pm 1.1$ & $185.7 \pm 6.8$ & $104.8 \pm 21.2$ \\
\hline
\end{tabular}

*significantly less than OL, DL, TE $(\mathrm{p}<0.05)$

${ }^{+}$significantly greater than all positions except OL, DL $(\mathrm{p}<0.05)$ 
Table 2. Mean \pm SD of ultrasound characteristics for position, race, year classification, and starter status subgroups.

\begin{tabular}{|c|c|c|c|}
\hline Group & N & mCSA $\left(\mathbf{c m}^{2}\right)$ & EI (a. u.) \\
\hline QB & 5 & $38.4 \pm 6.9$ & $59.9 \pm 7.6$ \\
RB & 4 & $41.3 \pm 1.7$ & $59.1 \pm 2.8$ \\
TE & 4 & $36.6 \pm 5.4$ & $65.8 \pm 2.7$ \\
LB & 7 & $37.5 \pm 5.3^{*}$ & $61.8 \pm 4.0$ \\
DB & 15 & $37.3 \pm 5.4^{*}$ & $58.7 \pm 7.6$ \\
PK & 3 & $34.2 \pm 6.0^{*}$ & $55.3 \pm 6.4$ \\
DL & 9 & $46.7 \pm 4.2$ & $54.5 \pm 6.1$ \\
OL & 11 & $42.0 \pm 5.5$ & $59.5 \pm 6.3$ \\
WR & 10 & $32.3 \pm 5.7^{+}$ & $61.0 \pm 7.0$ \\
& & & \\
Black & 36 & $39.7 \pm 6.7$ & $57.5 \pm 6.4$ \\
White & 25 & $36.8 \pm 5.8$ & $61.0 \pm 6.7$ \\
Other & 8 & $40.4 \pm 7.7$ & $61.8 \pm 4.7$ \\
& & & \\
Freshman & 7 & $38.0 \pm 5.9$ & $58.0 \pm 6.2$ \\
Sophomore & 32 & $39.0 \pm 7.0$ & $59.8 \pm 6.5$ \\
Junior & 20 & $37.4 \pm 6.5$ & $58.6 \pm 7.4$ \\
Senior & 9 & $41.1 \pm 6.1$ & $59.9 \pm 6.2$ \\
& & & \\
Starter & 22 & $40.5 \pm 7.2$ & $58.4 \pm 6.7$ \\
Non-Starter & 46 & $38.0 \pm 6.2$ & $59.7 \pm 6.6$ \\
\hline
\end{tabular}

*significantly less than DL $(\mathrm{p}<0.05)$

${ }^{+}$significantly less than OL $(\mathrm{p}<0.05)$ 
Table 3. Mean \pm SD of DXA body composition characteristics for position, race, year classification, and starter status subgroups.

\begin{tabular}{|c|c|c|c|c|c|}
\hline Group & N & Fat mass $(\mathbf{k g})$ & Lean mass (kg) & $\begin{array}{c}\text { Legs Lean } \\
\text { mass (kg) }\end{array}$ & \% fat \\
\hline QB & 5 & $17.3 \pm 1.9^{*}$ & $74.6 \pm 2.9^{*}$ & $25.4 \pm 1.9$ & $18.1 \pm 2.1+$ \\
RB & 4 & $13.1 \pm 3.2^{*}$ & $76.6 \pm 6.9^{*}$ & $27.1 \pm 1.7$ & $13.9 \pm 2.0^{*}$ \\
TE & 4 & $19.4 \pm 4.0 \mathrm{a}$ & $84.2 \pm 3.8^{*}$ & $29.0 \pm 1.3$ & $17.9 \pm 2.8$ \\
LB & 7 & $17.4 \pm 3.0^{*}$ & $81.2 \pm 4.4^{*}$ & $28.8 \pm 1.8^{*}$ & $17.0 \pm 2.9^{*}$ \\
DB & 15 & $12.4 \pm 2.5^{*}$ & $72.3 \pm 4.5^{* \#}$ & $24.7 \pm 2.4^{*}$ & $14.0 \pm 2.2^{*}$ \\
PK & 3 & $14.3 \pm 3.4^{*}$ & $68.4 \pm 3.3^{*}$ & $23.0 \pm 1.5^{*}$ & $16.6 \pm 3.9^{*}$ \\
DL & 9 & $29.1 \pm 4.8$ & $96.2 \pm 4.8^{*}$ & $32.2 \pm 2.0$ & $22.3 \pm 2.3$ \\
OL & 12 & $32.7 \pm 4.6$ & $96.6 \pm 6.8^{*}$ & $31.7 \pm 2.5$ & $24.4 \pm 2.2$ \\
WR & 10 & $12.5 \pm 2.4^{*}$ & $70.8 \pm 5.0^{*}$ & $24.7 \pm 2.4^{*}$ & $14.4 \pm 2.2^{*}$ \\
& & & & & \\
Black & 36 & $18.0 \pm 8.9$ & $80.9 \pm 11.1$ & $29.5 \pm 3.9$ & $16.6 \pm 4.8$ \\
White & 26 & $21.0 \pm 8.1$ & $80.5 \pm 12.5$ & $27.5 \pm 3.7$ & $19.3 \pm 4.0$ \\
Other & 8 & $21.4 \pm 9.6$ & $85.6 \pm 13.4$ & $27.8 \pm 3.5$ & $18.6 \pm 4.4$ \\
& & & & & \\
Freshman & 7 & $14.7 \pm 10.6$ & $80.4 \pm 14.5$ & $27.7 \pm 4.4$ & $17.5 \pm 5.9$ \\
Sophomore & 33 & $19.7 \pm 8.6$ & $80.8 \pm 11.3$ & $27.9 \pm 3.2$ & $18.1 \pm 4.8$ \\
Junior & 20 & $18.9 \pm 8.1$ & $81.4 \pm 12.5$ & $26.5 \pm 7.3$ & $17.5 \pm 4.1$ \\
Senior & 9 & $20.4 \pm 9.1$ & $83.6 \pm 11.8$ & $28.2 \pm 4.0$ & $17.9 \pm 4.4$ \\
& & & & & \\
Starter & 23 & $20.4 \pm 8.4$ & $83.3 \pm 11.8$ & $28.4 \pm 3.6$ & $18.2 \pm 4.3$ \\
Non-Starter & 46 & $18.4 \pm 8.8$ & $80.3 \pm 11.8$ & $27.1 \pm 5.5$ & $17.7 \pm 4.8$ \\
\hline
\end{tabular}

*significantly less than OL, DL

+significantly greater than $\mathrm{DB}$, significantly less than OL

a significantly less than OL only

\#significantly less than TE, LB 
Table 4. Correlations between US and DXA measures percent body fat (\%fat), lean mass (LM), fat mass (FM), muscle cross sectional area (mCSA), echo intensity (EI)

\begin{tabular}{|c|c|c|c|c|c|c|}
\hline & $\%$ fat & LM & FM & Legs LM & mCSA & EI \\
\hline$\%$ fat & . & $0.772 * *$ & $0.876^{* *}$ & $0.667 * *$ & $0.388 * *$ & -0.048 \\
\hline $\mathbf{L M}$ & & - & $0.809 * *$ & $0.953 * *$ & $0.611 * *$ & -0.213 \\
\hline FM & & & - & $0.722 * *$ & $0.424 * *$ & -0.075 \\
\hline Legs LM & & & & - & $0.632 * *$ & -0.227 \\
\hline mCSA & & & & & - & $-0.455^{*} *$ \\
\hline EI & & & & & & - \\
\hline
\end{tabular}

** Significant $(\mathrm{p}<0.01)$. 
Figure 1. Individual comparisons of height $(\mathrm{cm})$ and weight $(\mathrm{kg})$ stratified by player position.

\section{Height vs. Weight}

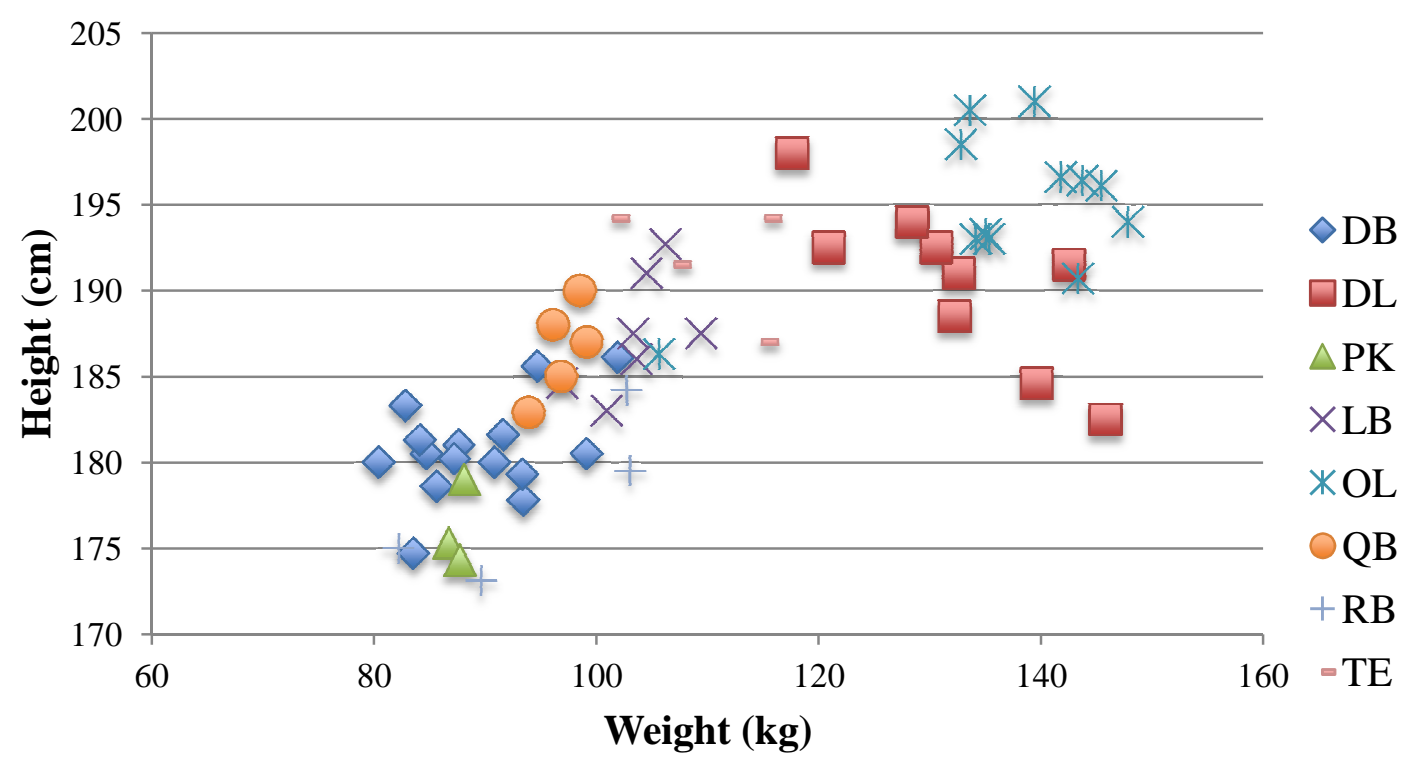


Figure 2. Individual echo intensity (a.u.) values stratified by race $(\mathrm{p}=0.063)$.

\section{Echo Intensity}

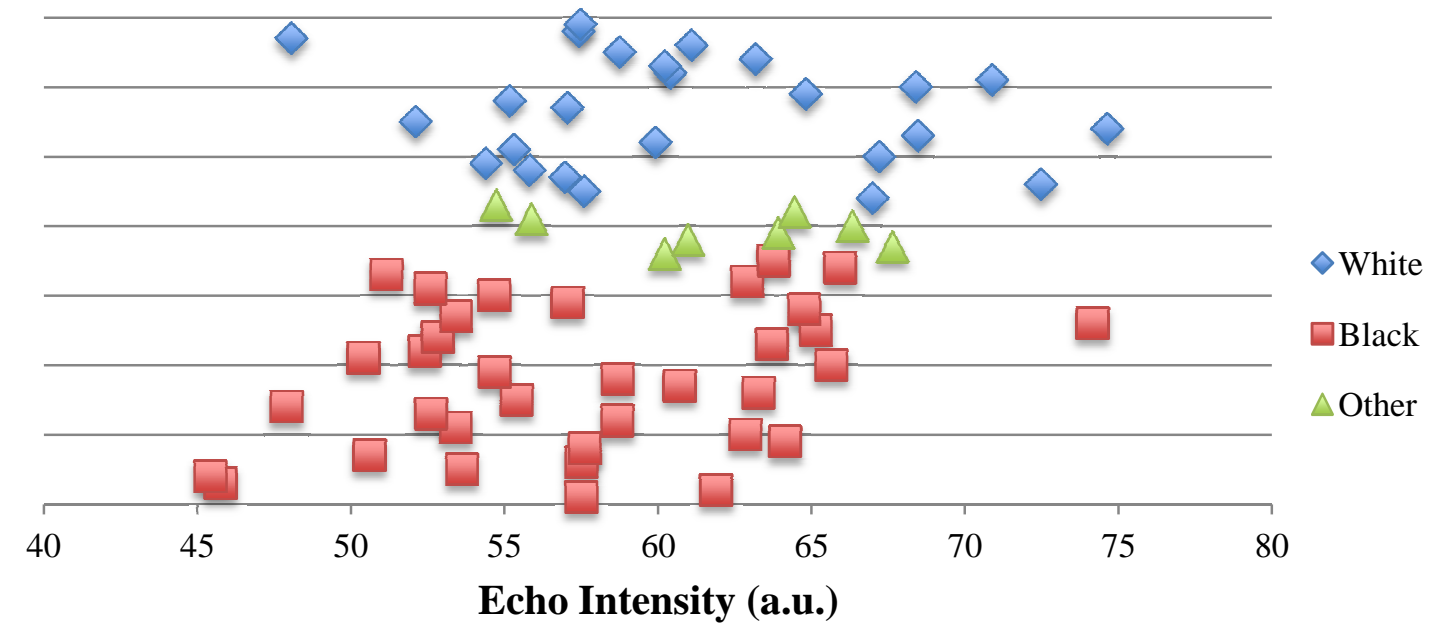


Figure 3. Individual comparisons of lean mass $(\mathrm{kg})$ and fat mass $(\mathrm{kg})$ stratified by player position.

\section{Lean Mass vs. Fat Mass}

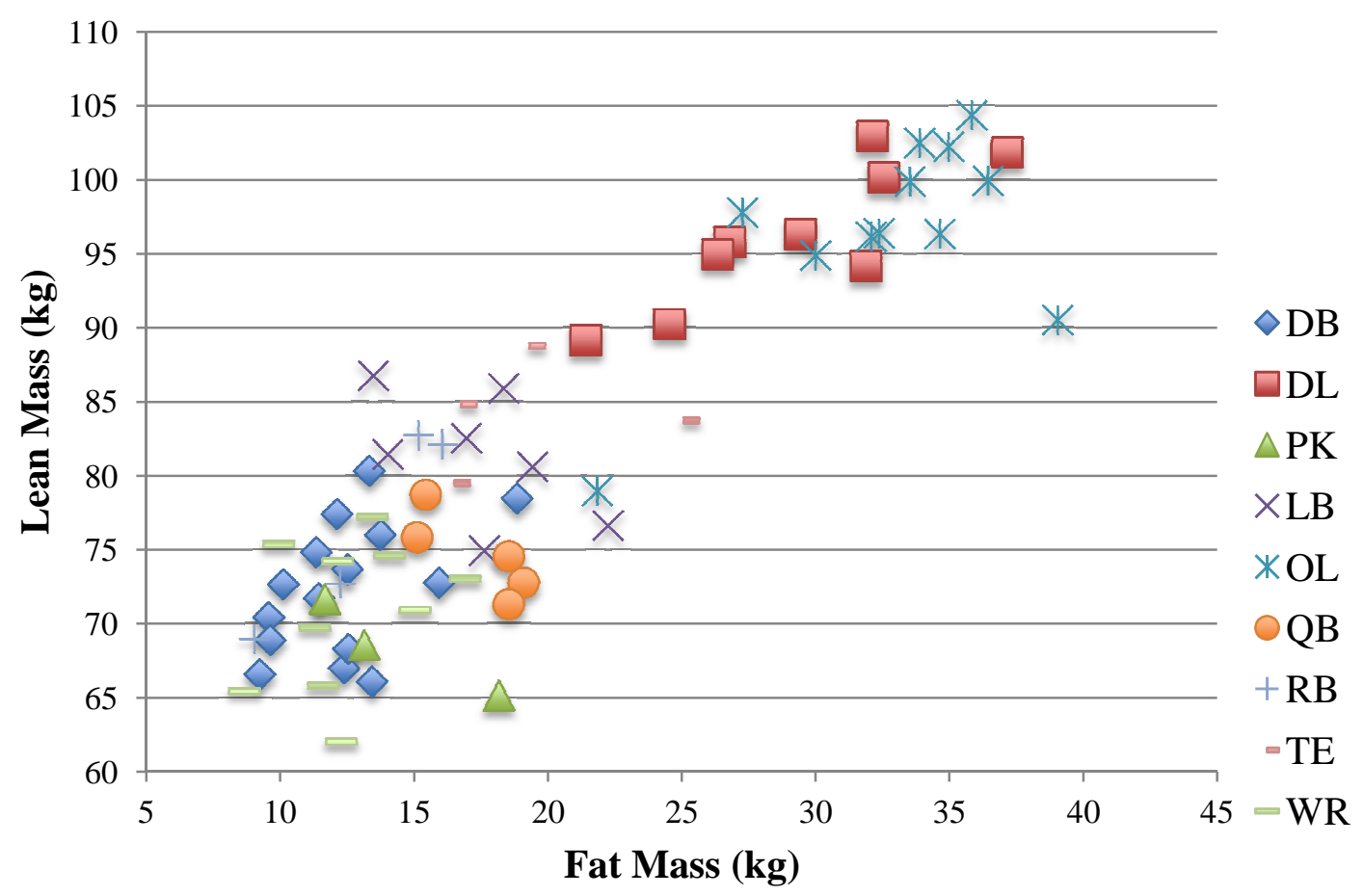


Figure 4. Individual percent fat values stratified by race $(\mathrm{p}=0.074)$.

\section{Percent Fat}

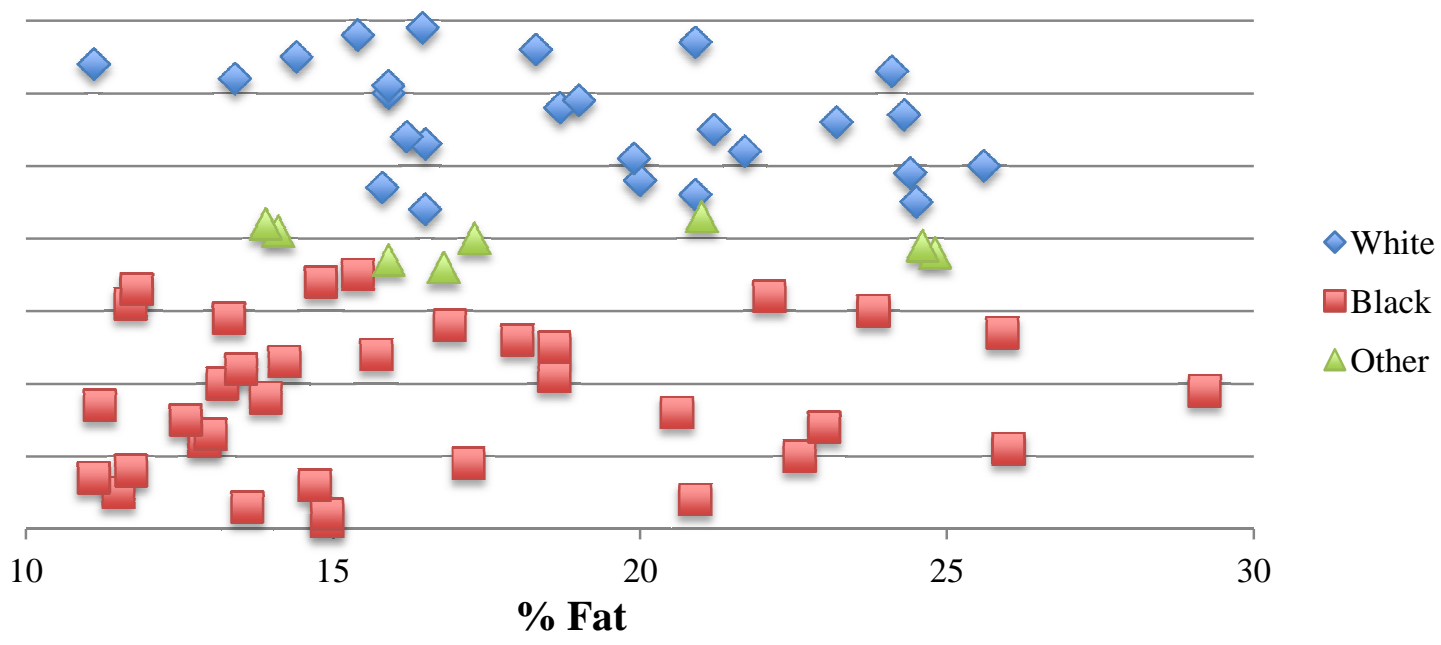




\section{REFERENCES}

1. Abe, T., Brown, J. B., \& Brechue, W. F. (1999). Architectural characteristics of muscle in black and white college football players. Medicine \& Science in Sports \& Exercise, 31, 1448-1452.

2. About Race. (2012). U.S. Census Bureau.

3. Achten, J., \& Jeukendrup, A. E. (2004). Optimizing fat oxidation through exercise and diet. Nutrition Burbank Los Angeles County Calif, 20, 716-727.

4. Ahtiainen, J. P., Hoffren, M., Hulmi, J. J., Pietikäinen, M., Mero, A. A., Avela, J., \& Häkkinen, K. (2010). Panoramic ultrasonography is a valid method to measure changes in skeletal muscle cross-sectional area. European Journal of Applied Physiology, 108, 273-279.

5. Albuquerque, F. N., Sert Kuniyoshi, F. H., Calvin, A. D., Sierra-Johnson, J., Romero Corral, A., Lopez-Jimenez, F., \& Somers, V. K. (2010). Sleep-disordered breathing, hypertension, and obesity in retired national football league players. Journal of the American College of Cardiology, 56(17), 1432-1433.

6. Ama, P. F., Simoneau, J. A., Boulay, M. R., Serresse, O., Thériault, G., \& Bouchard, C. (1986). Skeletal muscle characteristics in sedentary black and Caucasian males. Journal of Applied Physiology, 61, 1758-1761.

7. Arts, I. M. P., Pillen, S., Schelhaas, H. J., Overeem, S., \& Zwarts, M. J. (2010). Normal values for quantitative muscle ultrasonography in adults. Muscle \& Nerve, 4l(1), 32-41. doi:10.1002/mus.21458

8. Body Composition Data for Individuals 8 Years of Age and Older: U.S.Population, 1999-2004. (2010). CDC.

9. Bosy-Westphal, A., Later, W., Hitze, B., Sato, T., Kossel, E., Gluer, C.-C., Muller, M. J. (2008). Accuracy of bioelectrical impedance consumer devices for measurement of body composition in comparison to whole body magnetic resonance imaging and dual X-ray absorptiometry. Obesity Facts, 1, 319-324.

doi:10.1159/000176061

10. Cadore, E. L., Izquierdo, M., Conceição, M., Radaelli, R., Pinto, R. S., Baroni, B. M., Kruel, L. F. M. (2012). Echo intensity is associated with skeletal muscle power and cardiovascular performance in elderly men. Experimental Gerontology, 47, 473-8. doi:10.1016/j.exger.2012.04.002

11. Collins, M. A., Millard-Stafford, M. L., Sparling, P. B., Snow, T. K., Rosskopf, L. B., Webb, S. A., \& Omer, J. (1999). Evaluation of the BOD POD for assessing body fat 
in collegiate football players. Medicine and Science in Sports and Exercise, 31, 13501356. doi:10.1097/00005768-199909000-00019

12. Dengel, D., Bosch, T., Burruss, T., Fielding, K., Engel, B., Weir, N., \& Weston, T. (2014). Body Composition and Bone Mineral Density of National Football League Players, 28(1), 1-6.

13. Deschenes, M. R., \& Kraemer, W. J. (2002). Performance and physiologic adaptations to resistance training. American Journal of Physical Medicine \& Rehabilitation / Association of Academic Physiatrists, 81(11), S3-16. doi:10.1097/01.PHM.0000029722.06777.E9

14. Friedlander, A. L., Casazza, G. A., Horning, M. A., Buddinger, T. F., \& Brooks, G. A. (1998). Effects of exercise intensity and training on lipid metabolism in young women. The American Journal of Physiology, 275, E853-E863.

15. Friedlander, A. L., Casazza, G. A., Horning, M. A., Usaj, A., \& Brooks, G. A. (1999). Endurance training increases fatty acid turnover, but not fat oxidation, in young men. Journal of applied physiology (Bethesda, Md. $\square:$ 1985), 86, 2097-2105).

16. Frontera, W. R., Hughes, V. A., Fielding, R. A., Fiatarone, M. A., Evans, W. J., \& Roubenoff, R. (2000). Aging of skeletal muscle: a 12-yr longitudinal study. Journal of Applied Physiology (Bethesda, Md. $\square:$ 1985), 88, 1321-1326.

17. Fukumoto, Y., Ikezoe, T., Yamada, Y., Tsukagoshi, R., Nakamura, M., Mori, N., Ichihashi, N. (2011). Skeletal muscle quality assessed from echo intensity is associated with muscle strength of middle-aged and elderly persons. European Journal of Applied Physiology, 112, 1519-25. doi:10.1007/s00421-011-2099-5

18. Fukumoto, Y., Ikezoe, T., Yamada, Y., Tsukagoshi, R., Nakamura, M., Mori, N., Ichihashi, N. (2012). Skeletal muscle quality assessed from echo intensity is associated with muscle strength of middle-aged and elderly persons. European Journal of Applied Physiology, 112(4), 1519-25. doi:10.1007/s00421-011-2099-5

19. Goodpaster, B. H. (2001). Skeletal Muscle Lipid Content and Insulin Resistance: Evidence for a Paradox in Endurance-Trained Athletes. Journal of Clinical Endocrinology Metabolism, 86, 5755-5761. doi:10.1210/jc.86.12.5755

20. Goodpaster, B. H., Theriault, R., Watkins, S. C., \& Kelley, D. E. (2000). Intramuscular lipid content is increased in obesity and decreased by weight loss. Metabolism Clinical And Experimental, 49, 467-472. Retrieved from http://www.ncbi.nlm.nih.gov/pubmed/10778870

21. Gregg, E. W., Cadwell, B. L., Cheng, Y. J., Cowie, C. C., Williams, D. E., Geiss, L., Vinicor, F. (2004). Trends in the prevalence and ratio of diagnosed to undiagnosed 
diabetes according to obesity levels in the U.S. Diabetes Care, 27, 2806-2812. doi:10.2337/diacare.27.12.2806

22. Harp, J. B., \& Hecht, L. (2005). Obesity in the National Football League, 293(9), 1058-1062.

23. Hawley, J. A., Brouns, F., \& Jeukendrup, A. (1998). Strategies to enhance fat utilisation during exercise. Sports Medicine (Auckland, N.Z.), 25, 241-257. doi:10.2165/00007256-199825040-00003

24. Heyward, V. H. (2001). ASEP Methods recommendation: Body composition assessment. Journal of Exercise Physiology, 4(4), 1-12.

25. Hyman, M. H., Dang, D. L., \& Liu, Y. (2012). Differences in obesity measures and selected comorbidities in former National Football League professional athletes. Journal of Occupational and Environmental Medicine / American College of Occupational and Environmental Medicine, 54(7), 816-9. doi:10.1097/JOM.0b013e3182572e53

26. Ikezoe, T., Asakawa, Y., Fukumoto, Y., Tsukagoshi, R., \& Ichihashi, N. (2012). Associations of muscle stiffness and thickness with muscle strength and muscle power in elderly women. Geriatrics \& Gerontology International, 12(1), 86-92. doi:10.1111/j.1447-0594.2011.00735.x

27. Jacobson, B., Conchola, E., Glass, R., \& Thompson, B. (2013). Longitudinal Morphological and Performance Profiles for American, NCAA Division I Football Players, 27(9), 2347-2354.

28. Kelly, D. F., Chaloner, C., Evans, D., Mathews, A., Cohan, P., Swerdloff, R., Guskiewicz, K. (2014). Prevalence of pituitary hormone dysfunction, metabolic syndrome and impaired quality of life in retired professional football players: a prospective study. Journal of Neurotrauma, 1, 1-43.

29. Kopelman, P. (2007). Health risks associated with overweight and obesity. Obesity Reviews $\square$ : An Official Journal of the International Association for the Study of Obesity, 8 (11), 13-7. doi:10.1111/j.1467-789X.2007.00311.x

30. Kraemer, W., Torine, J., Silvestre, R., French, D. N., Ratamess, N., Spiering, B., Volek, J. (2005). Body Size and Composition of National Football Leage Players. Journal of Strength and Conditioning Research, 19(3), 485-490.

31. Lenz, M., Richter, T., \& Mühlhauser, I. (2009). The morbidity and mortality associated with overweight and obesity in adulthood: a systematic review. Deutsches Ärzteblatt International, 106, 641-8. doi:10.3238/arztebl.2009.0641 
32. Mathews, E., \& Wagner, D. R. (2008). Prevalence of Overweight and Obesity in Collegiate American Football Players, by Position. Journal Of American College Health, 57(1), 33-38.

33. Miljkovic, I., Cauley, J. A., Petit, M. A., Ensrud, K. E., Strotmeyer, E., Sheu, Y., ... Zmuda, J. M. (2009). Greater adipose tissue infiltration in skeletal muscle among older men of African ancestry. The Journal of Clinical Endocrinology \& Metabolism, 94, 2735-2742. doi:10.1210/jc.2008-2541

34. Miljkovic, I., \& Zmuda, J. M. (2010). Epidemiology of myosteatosis. Current Opinion in Clinical Nutrition and Metabolic Care, 13, 260-264.

35. Miller, M. A., Croft, L. B., Belanger, A. R., Romero-Corral, A., Somers, V. K., Roberts, A. J., \& Goldman, M. E. (2008). Prevalence of metabolic syndrome in retired National Football League players. The American Journal of Cardiology, 101, 1281-1284. doi:10.1016/j.amjcard.2007.12.029

36. Miller, T. a, White, E. D., Kinley, K. a, Congleton, J. J., \& Clark, M. J. (2002). The effects of training history, player position, and body composition on exercise performance in collegiate football players. Journal of Strength and Conditioning Research / National Strength \& Conditioning Association, 16(1), 44-9.

37. Moon, J. R., Hull, H. R., Tobkin, S. E., Teramoto, M., Karabulut, M., Roberts, M. D., Stout, J. R. (2007). Journal of the International Society of Sports Nutrition laboratory methods $\square$ : a three-compartment model approach, 9, 1-9. doi:10.1186/1550-2783-4-

38. Nieman, D. (2010). Exercise Testing and Prescription (7th ed.). New York, NY: McGraw-Hill.

39. Nijboer-Oosterveld, J. Nens Van Alfen, P., Pillen, P. (2011). New Nomal Values for Quantitative Muscle Ultrasound: Obesity Increases Muscle Echo Intensity. Muscle \& Nerve, 43(1), 142-3. doi:10.1002/mus.21842

40. Noel, M. B., VanHeest, J. L., Zaneteas, P., \& Rodgers, C. D. (2003). Body composition in Division I football players. Journal of Strength and Conditioning Research / National Strength \& Conditioning Association, 17(2), 228-37.

41. Norcross, J., \& Van Loan, M. D. (2004). Validation of fan beam dual energy x ray absorptiometry for body composition assessment in adults aged 18-45 years. British Journal of Sports Medicine, 38(4), 472-6. doi:10.1136/bjsm.2003.005413

42. Olson, D. E., Sikka, R. S., Hamilton, A., \& Krohn, A. (2011). Football Injuries: Current Concepts. Current Sports Medicine Reports, 10(5), 290-298.

43. Pateyjohns, I. R., Brinkworth, G. D., Buckley, J. D., Noakes, M., \& Clifton, P. M. (2006). Comparison of three bioelectrical impedance methods with DXA in 
overweight and obese men. Obesity (Silver Spring, Md.), 14, 2064-2070.

doi:10.1038/oby.2006.241

44. Pineau, J.-C., Guihard-Costa, A.-M., \& Bocquet, M. (2007). Validation of ultrasound techniques applied to body fat measurement: A comparison between ultrasound techniques, air displacement plethysmography and bioelectrical impedance vs. dualenergy X-ray absorptiometry. Annals of Nutrition \& Metabolism, 51, 421-427. doi:10.1159/000111161

45. Prior, B. M., Cureton, K. J., Modlesky, C. M., Evans, E. M., Sloniger, M. A., Saunders, M., \& Lewis, R. D. (1997). In vivo validation of whole body composition estimates from dual-energy X-ray absorptiometry. Journal of Applied Physiology (Bethesda, Md. $\square:$ 1985), 83, 623-630.

46. Robbins, D. W., Goodale, T. L., Kuzmits, F. E., \& Adams, A. J. (2012). Changes in the Athletic Profile of Elite College American Football Players. Journal of Strength and Conditioning Research, 27(4), 861-874. doi:10.1519/JSC.0b013e3182610be2

47. Roden, M. (2005). Muscle triglycerides and mitochondrial function: possible mechanisms for the development of type 2 diabetes. International Journal of Obesity (2005), 29(2), S111-S115.

48. Rosenberg, J. G., Ryan, E. D., Ph, D., Sobolewski, E. J., Michael, J., Thompson, B. J., \& King, G. E. (2013). Muscle \& Nerve, 1-22.

49. Sardinha, L. B., Lohman, T. G., Teixeira, P. J., Guedes, D. P., \& Going, S. B. (1998). Comparison of air displacement plethysmography with dual-energy X-ray absorptiometry and 3 field methods for estimating body composition in middle-aged men. The American Journal of Clinical Nutrition, 68, 786-793.

50. Secora, C. A., Latin, R. W., Berg, K. E., \& Noble, J. M. (2004). Comparison of physical and performance characteristics of NCAA Division I football players: 1987 and 2000. Journal Of Strength \& Conditioning Research, 18(2), 186-291.

51. Selinger, A. (1977). The body as a three component system. University of Illinois, Urbana.

52. Sipila, S., \& Suominen, H. (1991). Ultrasound Imaging of the Quadriceps Muscle in Elderly, Muscle \& Nervce, 14(6) 527-533.

53. Sipilä, S., \& Suominen, H. (1993). Muscle ultrasonography and computed tomography in elderly trained and untrained women. Muscle \& Nerve, 16, 294-300. doi:10.1002/mus.880160309

54. Siri, W. E. (1961). Body composition from fluid spaces and density. (B. J. HA, Ed.). Washington DC. 
55. Snow, T., Miller-Stafford, M., \& Rosskopf, L. B. (1998). Body Composition Profile of NFL Football Players. Journal of Strength and Conditioning Research, 12(3), 146149.

56. Tracy, B. L., Ivey, F. M., Hurlbut, D., Martel, G. F., Lemmer, J. T., Siegel, E. L., Hurley, B. F. (1999). Muscle quality . II . Effects of strength training in 65- to 75-yrold men and women. Journal of Applied Physiology, (86), 195-201.

57. Van Aggel-Leijssen, D. P. C., Saris, W. H. M., Wagenmakers, A. J. M., Senden, J. M., \& van Baak, M. A. (2002). Effect of exercise training at different intensities on fat metabolism of obese men. Journal of applied physiology (Bethesda, Md. $\square:$ 1985), 92, 1300-1309). doi:10.1152/japplphysiol.00030.2001

58. Van Loan, M. D., \& Mayclin, P. L. (1992). Body composition assessment: dualenergy X-ray absorptiometry (DEXA) compared to reference methods. European Journal of Clinical Nutrition, 46, 125-130.

59. Wagner, D. R., Heyward, V. H., \& Gibson, A. L. (2000). Validation of air displacement plethysmography for assessing body composition. Medicine and Science in Sports and Exercise, 32, 1339-1344. doi:10.1097/00005768-20000700000023

60. Wang, Z., Heymsfield, S. B., Chen, Z., Zhu, S., \& Pierson, R. N. (2010). Estimation of percentage body fat by dual-energy $\mathrm{x}$-ray absorptiometry: evaluation by in vivo human elemental composition. Physics in Medicine and Biology, 55(9), 2619-35. doi:10.1088/0031-9155/55/9/013

61. Wang, Z. M., Deurenberg, P., Guo, S. S., Pietrobelli, a, Wang, J., Pierson, R. N., \& Heymsfield, S. B. (1998). Six-compartment body composition model: inter-method comparisons of total body fat measurement. Journal of the International Association for the Study of Obesity, 22(4), 329-37.

62. What is average NFL player's career length? Longer than you might think, Commissioner Goodell says. (2011). NFL Communications. Retrieved from http://nflcommunications.com/2011/04/18/what-is-average-nfl-player\%E2\%80\%99scareer-length-longer-than-you-might-think-commissioner-goodell-says/

63. World Health Organization. (2013). Obesity and overweight. WHO Media Centre. Retrieved from http://www.who.int/mediacentre/factsheets/fs311/en/\# 